\title{
AMERICAN
WATER RESOURCES
}

\section{Improving U.S. National Water Model Streamflow with Long Short-Term Memory Networks}

\begin{tabular}{|r|l|}
\hline Journal: & Journal of the American Water Resources Association \\
\hline Manuscript ID & JAWRA-20-0099-P.R1 \\
\hline Manuscript Type: & Technical Paper \\
\hline Author: & n/a \\
\hline Complete List of Authors: & $\begin{array}{l}\text { Frame, Jonathan; University of Alabama, Geological Sciences } \\
\text { Nearing, Grey; Google Research } \\
\text { Kratzert, Frederik; Johannes Kepler University, LIT AI Lab \& Institute for } \\
\text { Machine Learning } \\
\text { Raney, Austin; University of Alabama, Geography } \\
\text { Rahman, Mashrekur; University of Alabama, Geological Sciences } \\
\text { Salas, Fernando R.; NOAA, NWS Office of Water Prediction }\end{array}$ \\
\hline Category Headings: & HYDROLOGY, MODELING \\
\hline Key Terms: & $\begin{array}{l}\text { streamflow < HYDROLOGY, National Water Model, long short-term } \\
\text { memory, post-processing, machine learning }\end{array}$ \\
\hline &
\end{tabular}




\section{Improving U.S. National Water Model Streamflow with Long Short-Term Memory} Networks

Jonathan M. Frame, Grey S. Nearing, Frederik Kratzert, Austin Raney, Mashrekur Rahman and

Fernando Salas

5 Department of Geological Sciences, University of Alabama, Tuscaloosa, AL USA (Frame \&

6 Rahman); Google Research, Mountain View, CA USA (Nearing); LIT AI Lab \& Institute for

7 Machine Learning, Johannes Kepler University, Linz, Austria (Kratzert); Department of

8 Geography, University of Alabama; Tuscaloosa, AL USA (Raney); NOAA National Water

9 Center (Salas).

10 (Correspondence to Frame: jmframe@crimson.ua.edu)

11 Research Impact Statement: Post-processing the U.S. National Water Model (NWM) with 12 deep learning improves streamflow predictions and identified the NWM channel router as a 13 major source of information loss.

14 ABSTRACT: Long short-term memory (LSTM) deep learning networks were used to post15 process the U.S. National Water Model (NWM) outputs for improved daily averaged streamflow 16 predictions at 531 basins across the continental United States (CONUS). We compared post17 processed streamflow against the NWM and a baseline LSTM without NWM outputs. The 18 LSTM post-processors perform better, on average, than the NWM. Overall median NSE scores 19 are 0.62 for the NWM, 0.74 for the standalone LSTM and 0.73 and 0.75 for the two post20 processors. The LSTM with NWM inputs was not significantly better than a standalone LSTM, 21 indicating that the NWM provides only situational benefit for LSTM streamflow prediction. 22 Accuracy of predictions in 2 of the 531 basins was severely reduced by post-processing during 23 tests on ungauged basins, and we found no way to identify ahead of time (without streamflow 24 observations and predictions for comparison) basins where this might occur. The baseline LSTM 25 performs well in ungauged basins. The post-processor improves NWM streamflow predictions in 26 all regions within CONUS. A sensitivity analysis was used to diagnose the land surface 27 component of the NWM as the source of mass bias error and the channel router as a source of 28 simulation timing error. Our assessment indicates that the NWM routing scheme should be 29 considered a priority for NWM improvement. 
30 (KEYWORDS: National Water Model; theory-guided machine learning; long short-term 31 memory; streamflow; model diagnostics.) 
The U.S. National Water Model (NWM), based on WRF-Hydro (Cosgrove et al., 2015),

34 is an emerging large-scale hydrology simulator. Some specific details of the NWM

35 advancements in large scale hydrology are described by Elmer (2019, page 11), including

36 increased resolution and number of stream reaches (2.7 million) for a model covering the

37 continental United States (CONUS). A purported strength of WRF-Hydro is simulating

38 hydrologic dynamics, and specifically timing of hydrological response (Salas et al., 2018). The

39 predictive performance of the NWM (ability to match streamflow observations) has been shown

40 to vary widely. Hansen et al. (2019) evaluated the performance of the NWM in the Colorado

41 River Basin in terms of drought and low flows; they found better performance in the Upper

42 Colorado River Basin than in the Lower Colorado River Basin, and attributed this discrepancy to

43 the NWM's ability to simulate snowpack. WRF-Hydro has generally poor performance in the

44 Southwest and Northern Plains (Salas et al., 2018). Salas et al., 2018 hypothesized that error in

45 WRF-hydro might come from lakes, reservoirs, floodplain dynamics and soil parameter

46 calibration.

47 The NWM version 2.0 was calibrated at 1,457 basins within the CONUS domain. As a

48 point of comparison, the United States Geological Survey (USGS) records daily streamflow at

4928,529 basins (https://nwis.waterdata.usgs.gov/nwis, accessed June 2020). Calibrating the model

50 at each stream gauge within the NWM domain is a large computational expense, and while

51 regionalization strategies can be used to improve forecast accuracy without having to calibrate

52 each individual basin, accuracy typically suffers compared to direct calibration. Due to these

53 reasons and others, making accurate hydrological predictions over large scales is a challenging 
54 problem, however there are promising results in the machine learning and data science

55 communities that may be directly applicable to improving the NWM.

56 Machine learning (ML) is a powerful tool for hydrological modeling, and there has been

57 a call to merge ML with traditional hydrological modeling (Reichstein et al., 2019; Nearing et

58 al., 2020). One example of an ML approach that has been effective for hydrological prediction is

59 the "long short-term memory" network (LSTM) (Hochreiter, 1991; Hochreiter and Schmidhuber,

60 1997). The LSTM is a time series deep learning method that is particularly well suited to model

61 hydrologic processes because it mimics in certain ways the Markovian input-state-ouput

62 structure of a dynamical system (Kratzert et al., 2018). LSTMs have been effective at simulating

63 predictions of surface runoff at the daily time scale (Kratzert et al., 2019a), including in

64 ungauged catchments where traditional methods of calibration do not work (Kratzert et al.,

65 2019b), and also at sub-daily (hourly) timescales (Gauch et al., 2020). One potential problem

66 with ML, however, is that it lacks a physical basis. While there are emerging efforts in hydrology

67 to merge physical understanding with machine learning (Karpatne et al., 2017a; Daw et al.,

68 2020; Pelissier et al., 2019; Chadalawada et al., 2020; Tartakovsky et al., 2020, Read et al.,

69 2019; Nearing et al., 2020; Hoedt et al., 2021), the field of theory-guided machine learning

70 (Karpatne et al., 2017b) is still relatively immature in hydrology.

The NWM informs forecasts of many hydrologic conditions, including river ice,

72 snowpack, soil moisture and inundation, which are used for management applications such as

73 transportation, recreation, agriculture and fisheries (NOAA 2019). When ML is to be used in the

74 NWM it should not disrupt the delivery of these hydrologic forecasts, therefore an ML prediction

75 for streamflow that does not also include predictions of the other hydrologic states and variables

76 must be run in parallel with the existing process-based hydrologic model. A natural question 
77 arises: does the existing NWM formulation benefit the already highly accurate LSTM predictions

78 of streamflow?

79 Hydrologic post-processing can remove systematic errors in the model prediction, and

80 has been shown to improve forecast accuracy of both calibrated and uncalibrated basins,

81 particularly in wet basins (Ye et al., 2014). The general methodology of post-processing involves

82 taking the output of a process-based model and feeding it into a data-driven model. In this paper

83 we applied a LSTM-based post-processor for the NWM to improve basin-scale streamflow

84 predictions. This is a straightforward theory-guided machine learning approach. We tested a

85 post-processor that uses dynamic information only from the NWM outputs and compared the

86 results against the NWM itself. We also tested a post-processor that included both the NWM

87 outputs and NLDAS atmospheric forcings as inputs and compared against a 'baseline' LSTM

88 model trained only with atmospheric forcings (no NWM outputs).

We applied the LSTM post-processors to 531 basins across the CONUS. The basins

90 chosen for this large-scale analysis are mostly headwater catchments without engineered control

91 structures, such as dams, canals, and levees. This was a deliberate choice made for the purpose of

92 simulating a close-to-natural rainfall-runoff response. Our goal was to use the post-processor to

93 learn systematic corrections to simulated basin-scale rainfall-runoff processes that can improve

94 forecasts of streamflow, rather than the hydraulic engineering implications resulting from

95 simulated controlled flow, e.g., a reservoir release. Kim et al. (2020) showed the limitation of the

96 NWM to predict streamflow in a highly engineered watershed and the need for representing

97 controlled releases. Thus, we are using some of the simplest, and top performing, applications of

98 the NWM for these experiments. 
100

101

102

103

104

105

106

107

108

109

110

111

112

113

114

115

116

117

118

119

120

121

122

Data \& Models

CAMELS Catchments. This study used the Catchment Attributes and Meteorological dataset for Large Sample Studies (CAMELS) (CAMELS; Newman et al., 2015; Addor et al., 2017). These data were curated by the US National Center for Atmospheric Research (NCAR; https://ral.ucar.edu/solutions/products/camels, accessed March 2020), and we used the 531 (out of 671) basins that were chosen by Newman et al. (2015) for model benchmarking. Newman et al (2015) excluded basins with large discrepancies in different methods for measuring basin area and also basins larger than 2,000 $\mathrm{km}^{2}$. CAMELS data include corresponding daily streamflow records from USGS gauges, and meteorological forcing data (precipitation, $\mathrm{max} / \mathrm{min}$ temperature, vapor pressure and total solar radiation) come from North American Land Data Assimilation System (NLDAS; Xia et al., 2012).

National Water Model. We used the National Water Model version 2.0 reanalysis, which contains output from a 25-year (January 1993 through December 2019) retrospective simulation (https://docs.opendata.aws/nwm-archive/readme.html, accessed June 2020). The NWM retrospective ingests rainfall and other meteorological forcings from atmospheric reanalyses (https://water.noaa.gov/about/nwm, accessed June 2020.). NWM reanalysis output includes channel outputs (point fluxes: CHRT) and land surface (gridded states and fluxes:

LDAS \& RT) outputs. The specific features that we used from the NWM reanalysis are shown in Table 1. To be compatible with the LSTM model, which uses a one-day timestep and was trained using all basins simultaneously, we took the mean values of these model outputs across UTC calendar days (12AM - 11PM) to produce daily records from the hourly NWM when used as input to the LSTM, but for NWM streamflow diagnostics we used the local calendar day (based on U.S. time zone) to be compatible with the USGS gauge records. Channel routing point data 
123 (CHRT) were collected at each individual NWM stream reach that corresponds to the stream

124 gauge associated with each CAMELS catchment. Gridded land surface data (LDAS) was

125 collected from each $1 \mathrm{~km}^{2}$ Noah-MP cell contained within the boundaries of each CAMELS

126 catchment, and these were averaged to produce a single representative (lumped) value for each

127 catchment. Gridded routing data (RT) were collected from each $250 \mathrm{~m}^{2}$ cell, and we included the

128 mean and maximum value within the catchment boundary. We did not include lake input and

129 output fluxes because these would be inconsistent across basins (some basins have zero and

130 some basins have multiple lakes). Note that the units of the NWM outputs are not required for

131 the LSTM post-processor.

TABLE 1. National Water Model Output Data

\begin{tabular}{llll}
\hline & & \multicolumn{2}{l}{ NWM model } \\
Feature name & Feature & component & Resolution \\
\hline ACCET & Accumulated evapotranspiration & LDAS & $1 \mathrm{Km}$ \\
FIRA & Total net long-wave (LW) radiation to atmosphere & LDAS & $1 \mathrm{Km}$ \\
FSA & Total absorbed short-wave (SW) radiation & LDAS & $1 \mathrm{Km}$ \\
FSNO & Snow cover fraction on the ground & LDAS & $1 \mathrm{Km}$ \\
HFX & Total sensible heat to the atmosphere & LDAS & $1 \mathrm{Km}$ \\
LH & Latent heat to the atmosphere & LDAS & $1 \mathrm{Km}$ \\
SNEQV & Snow water equivalent & LDAS & $1 \mathrm{Km}$ \\
SNOWH & Snow depth & LDAS & $1 \mathrm{Km}$ \\
SOIL M (4 layers) & Volumetric soil moisture & LDAS & $1 \mathrm{Km}$ \\
SOIL W (4 layers) & Liquid volumetric soil moisture & LDAS & $1 \mathrm{Km}$ \\
TRAD & Surface radiative temperature & LDAS & $1 \mathrm{Km}$ \\
UGDRNOFF & Accumulated underground runoff & LDAS & $1 \mathrm{Km}$ \\
streamflow & River Flow & CHRT & point \\
q_lateral & Runoff into channel reach & CHRT & point \\
velocity & River Velocity & CHRT & point \\
qSfcLatRunoff & Runoff from terrain routing & CHRT & point \\
qBucket & Flux from groundwater bucket & CHRT & point \\
qBtmVertRunoff & Runoff from bottom of soil to groundwater bucket & CHRT & point \\
Sfcheadsubrt (mean and max) & Ponded water depth & RTOUT & $250 \mathrm{Km}$ \\
Zwattablrt (mean and max) & Water table depth & RTOUT & $250 \mathrm{Km}$
\end{tabular}


Long short-term memory network. The LSTM is a recurrent neural network that is able

135 to maintain a memory of the system state and dynamics through a period of time (in this case 365

136 days). This recurrent state space is the main advantage for hydrological applications over other

137 types of neural networks. Our LSTM network was developed from Kratzert et al. (2019) using a

138 codebase that is now referred to as NeuralHydrology (https://neuralhydrology.github.io/ accessed

139 March 2021). This research grade codebase was developed in the Python programming language

140 and is based primarily on the Pytorch machine learning library.

The LSTM used two types of inputs: daily meteorological forcings and static catchment

142 attributes. Again, note that the units of the forcing data are irrelevant when used as inputs for the

143 LSTM, which does not include a mass or energy balance. We normalized all inputs to the LSTM,

144 including static and dynamic inputs by subtracting the mean and dividing by the standard

145 deviation of the training data. We used eighteen catchment attributes from the CAMELS dataset

146 related to climate, vegetation, topography, geology, and soils. These are described in more detail

147 by Addor et al. (2017) and listed here in Table 2. Catchment attributes are static for each basin

148 (do not change in time). For the post-processing runs we added the states, fluxes, and streamflow

149 predictions from version 2.0 of the NWM.

TABLE 2. LSTM Inputs

\begin{tabular}{ll}
\hline \multicolumn{2}{c}{ Meteorological Forcing Data (used in models denoted with an "A") } \\
\hline Maximum Air Temp (TMax) & 2-meter daily maximum air temperature \\
Minimum Air Temp (TMin) & 2-meter daily minimum air temperature \\
Precipitation (PRCP) & Average daily precipitation \\
Radiation (SRAD) & Surface-incident solar radiation \\
Vapor Pressure (Vp) & Near-surface daily average \\
\hline \multicolumn{2}{c}{ Static Catchment Attributes (used in each of the LSTM models) } \\
\hline Precipitation Mean & Mean daily precipitation \\
PET Mean & Mean daily potential evapotranspiration \\
Aridity Index & Ratio of Mean PET to Mean Precipitation \\
& Estimated by representing annual precipitation and temperature as sin waves \\
Precipitation Seasonality & Positive (negative) values indicate precipitation peaks during the summer (winter).
\end{tabular}


2

3

4

5

6

7

8

9
Values of approx. 0 indicate uniform precipitation throughout the year.

Snow Fraction

Fraction of precipitation falling on days with temp [C].

Frequency of days with $\leq 5 \mathrm{x}$ mean daily precipitation. Average duration of high precipitation events (number of consecutive days with $\leq 5 \mathrm{x}$ mean daily

High Precipitation Frequency precipitation).

Low Precipitation Frequency

Frequency of dry days $(<1 \mathrm{~mm} /$ day $)$.

Average duration of dry periods (number of consecutive days with precipitation $<1$

Low Precipitation Duration $\mathrm{mm} /$ day).

Elevation

Catchment mean elevation.

Slope

Area

Forest Fraction

LAI Max

LAI Difference

GVF Max

GVF Difference

Soil Depth (Pelletier)

Catchment mean slope.

Catchment area.

Fraction of catchment covered by forest.

Maximum monthly mean of leaf area index.

Difference between the max. and min. mean of the leaf area index.

Maximum monthly mean of green vegetation fraction.

Difference between the maximum and minimum monthly mean of the green vegetation fraction.

Depth to bedrock (maximum 50m).

Soil depth (maximum $1.5 \mathrm{~m}$ ).

Volumetric porosity.

Saturated hydraulic conductivity.

Maximum water content of the soil.

Fraction of sand in the soil.

Fraction of silt in the soil.

Fraction of clay in the soil.

Fraction of the catchment area characterized as "carbonate sedimentary rocks".

Surface permeability $(\log 10)$.

We trained the LSTM models to make predictions at all 531 CAMELS catchments used

153 in the analysis. We split the data temporally into a training period and testing period, and we

154 present no results from the training period as these results are unrepresentative of the out-of-

155 sample predictions. We trained the LSTMs on water years 2004 through 2014 and tested on

156 water years 1994 through 2002. No spatial splits were included in the training procedure. The

157 LSTMs used a 365-day LSTM look-back period, so a full year gap was left between training and

158 testing to prevent bleedover (i.e., information exchange) between the two periods. We trained

159 separate LSTMs with ten unique random seeds for initializing weights and biases, and calculated

160 benchmarking statistics using the ensemble mean hydrograph. The LSTMs make predictions 
161 representing runoff in units $[\mathrm{mm}]$, reflecting an area normalized volume of water that moves

162 through a stream at each model timestep. USGS gauge records (and the NWM predictions) are in

163 streamflow units $\left[\mathrm{L}^{3} / \mathrm{T}\right]$. We used the geospatial fabric estimate of the catchment area provided

164 in the CAMELS dataset to convert all streamflow to units [L] for our diagnostic comparison. We

165 trained the LSTMs with the protocol and features described in Appendix B of Kratzert et al.

166 (2019b): this includes 30 epochs, a hyperbolic tangent activation function, a hidden layer size of

167256 cell states, a look-back of 365 days, variable learning rates set at epoch 0 to 0.001 , epoch 11

168 to 0.005 and epoch 21 to 0.0001 , dropout rate of 0.4 and an input sequence length: 270 .

\section{Experimental Design}

170 We tested the results from LSTM post-processing against the NWM and also against a

171 baseline LSTM with no inputs from the NWM (referred to as LSTM_A, in which the A stands

172 for atmospheric forcing). Table 3 will guide the reader through the setup of each model.

TABLE 3. Models

\begin{tabular}{lcl}
\hline Model label & $\begin{array}{c}\text { Number of dynamic LSTM } \\
\text { inputs }\end{array}$ & \multicolumn{1}{c}{ Model description } \\
\hline NWM & N/A & National Water Model mean daily streamflow predictions \\
LSTM_PP & 28 & LSTM trained with NWM output for post processing \\
LSTM_PPA & 32 & LSTM trained with NWM output and atmospheric forcings for post- \\
& 5 & processing \\
LSTM_A & 5 & LSTM trained with atmospheric forcing conditions.
\end{tabular}

176 post-processors (LSTM_PP \& LSTM_PPA) used NWM outputs as LSTM inputs, and the results 177 were LSTM-based streamflow predictions influenced by the process-based NWM. This is a 178 straightforward method of theory-guided machine learning. 
LSTM-A

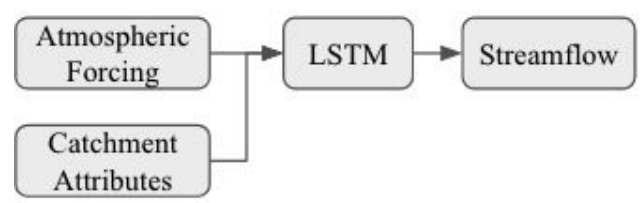

LSTM-PP

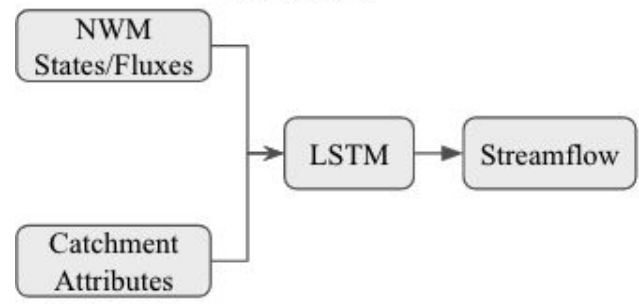

\section{LSTM-PPA}

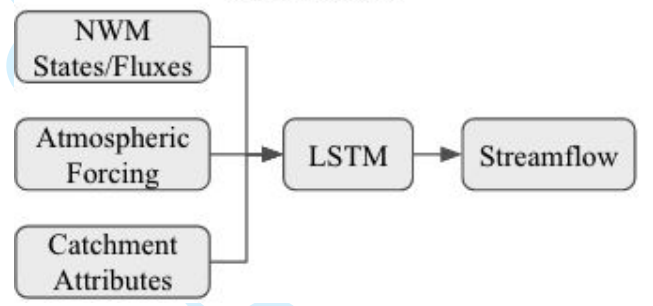

180 FIGURE 1. Flow chart showing the baseline LSTM (LSTM_A) and the LSTM post-processors with NWM data as 181 inputs (LSTM_PP \& LSTM_PPA). LSTM_PP is the post-processor which used only NWM outputs as input to an 182 LSTM, and LSTM_PPA used both the NWM outputs and atmospheric forcings.

183 As a quality check, we compared the results from each LSTM ensemble member, and 184 found a relative standard error of the mean streamflow about $1 \%$, and relative standard error of 185 the Nash-Sutcliffe Efficiency (NSE) value of about $0.01 \%$. This means that all LSTM solutions 186 are similar between random initialization seeds. Gauch et al. (2019) attributed a 0.01 discrepancy 187 in NSE values of the LSTM predictions to non-determinism of the loss function minimization. In 188 our experiments discrepancies in the loss function occur between different random seed 189 initializations, but running the training procedure twice with the same random seed gives an 190 identical solution, satisfying the definition of determinism. 
Performance metrics. We calculated several metrics to evaluate predictive performance,

192 including the NSE and Kling-Gupta Efficiency (KGE) values (Gupta et. al, 2009). The variance,

193 bias and Pearson correlation metrics were calculated separately as components of the NSE

194 (Gupta et al., 2009); these tell us about relative variability, mass conservation and linear

195 correlation between the modeled/observed streamflow values, respectively. Observed streamflow

196 values are from the USGS streamflow gauges associated with each of the CAMELS basins. The

197 metrics were calculated in two ways: 1) at each basin and then averaged together, and 2) using

198 all of the flows from all basins combined.

199 Our graphical results focus on three performance metrics: (i) NSE measures the overall

200 predictive performance as a correlation coefficient for the 1:1 linear fit between simulations and

201 observations, (ii) Peak timing error measures the absolute value of differences (in units days)

202 between simulated and observed peak flows for a given event, and (iii) total (absolute) bias

203 measures the overall bias of the simulated hydrograph relative to observations and represents

204 how well the model matches the total volume of partitioned rainfall that passes through the

205 stream gauge at each basin.

206 We also calculated performance metrics on different flow regimes. Rising limbs and

207 falling limbs were characterized by a one-day derivative, where positive derivatives were

208 categorized as rising limb, and negative derivatives as falling limb. High flows were

209 characterized as all flow above the $80^{\text {th }}$ percentile in a given basin, and low flows as below the

$21020^{\text {th }}$ percentile in a given basin.

211 We tested the performance of the LSTM post-processors in different regions. We split the 212 basins by USGS designated "water resource regions" (https://water.usgs.gov/GIS/regions.html, 
213 accessed July 2020). To analyze the regions individually we averaged the NSE, bias and timing

214 error of the CAMELS basins within each region.

215 We set an alpha value for statistical significance to $\alpha=0.05$. To control for multiple

216 comparisons we adjusted the alpha values using family-wise error rate equal to $1-(1-\alpha)^{\mathrm{m}}$, with $\mathrm{m}$

217 being the number of significance tests (86 in total), which brought our effective alpha value

218 down to 0.049. We tested for statistical significance with a Wilcoxon signed-rank test against the

219 null hypothesis that our test models (LSTM post-processors) performance across basins came

220 from the same distribution as our base models (NWM \& LSTM_A).

221 Simulated hydrograph representation of hydrologic signatures. Hydrologic

222 signatures help us understand how well a model represents important aspects of real-world

223 streamflow, and where improvement should be made to the model's conceptualization (Gupta et

$224 a l ., 2008)$. We analyzed the hydrologic signatures described by Addor et al. (2018), and these are

225 listed below in Table 4. We calculated the true signatures with USGS streamflow observations,

226 and calculated model representations with predicted values of daily streamflow. The comparison

227 between true values and predicted values was made with a correlation coefficient $\left(\mathrm{r}^{2}\right)$ across

228 basins (one value of the observed and predicted hydrologic signatures were calculated per basin),

229 higher values indicate better representation of hydrologic signature across basins by the model.

230 We used the Steiger method to test for statistically significant improvement (or detriment)

231 between the base models and the LSTM post-processor (Steiger and Browne, 1984).

TABLE 4. Hydrologic signatures (adapted from Addor et al. 2018)

\begin{tabular}{lr}
\hline Signature description & Signature name \\
\hline Average duration of low-flow events & low_q_dur \\
Frequency of days with zero flow & zero_q_freq \\
Average duration of high-flow events & high_q_dur \\
Streamflow precipitation elasticity & stream_elas
\end{tabular}




Frequency of high-flow days
Slope of the flow duration curve
Frequency of low-flow days
Baseflow index
Runoff ratio
Mean half-flow date
5 percent flow quantile
95 percent flow quantile
Mean daily discharge

Identifying basins best suited for post-processing with multi-linear regression. The

235 LSTM post-processors did not improve performance at every basin. It therefore would be

236 valuable to know if a LSTM post-processor will work in any particular basin before

237 implementation. We trained a multi-linear regression, using the Scikit-learn library in Python, to

238 predict the performance changes between the NWM and the LSTM post-processors (LSTM_PP

239 \& LSTM_PPA) at each individual basin. The inputs to the regression analysis were the

240 performance score of the NWM streamflow predictions, hydrologic signatures and catchment

241 characteristics. These regressors are useful to help interpret what basins might benefit most from

242 an LSTM post-processor. We trained and tested multi-linear regression models using k-fold

243 cross-validation with 20 splits $(\mathrm{k}=20)$ over the 531 basins. We report the correlation $\left(\mathrm{r}^{2}\right)$ of out-

244 of-sample regression predictions of post-processing improvements vs. actual post-processing

245 improvements.

Interpretation of LSTM with integrated gradients. We aim to explain the relationship

247 between a model's predictions in terms of its features. This will help us understand feature

248 importance, identifying data issues, and inform NWM process diagnostics from the post-

249 processors. We calculated integrated gradients (Sundararajan et al., 2017) to attribute the LSTM 250 inputs (both atmospheric forcings and NWM outputs) to the total prediction of streamflow. 
251 Integrate gradients are a type of sensitivity analysis that are relatively insensitive to low gradients

252 (e.g., at the extremes of neural network activation functions). We calculated integrated gradients

253 separately for each input, at each timestep, for each lookback timestep, in each basin. This means

254 that for 9 years of test data with a 365-day lookback there were about 1.2 million integrated

255 gradients per input, per basin. The unit of the integrated gradient is technically normalized

256 streamflow, but we were mostly interested in the relative values of integrated gradients of each

257 individual LSTM input.

258 Interpretation of LSTM with correlations between performance and NWM inputs.

259 We made a direct connection between LSTM post-processor improvements with the NWM 260 outputs using correlation. We calculated Pearson $\mathrm{R}$ values between the basin average value of 261 each NWM input feature and the total performance change (NSE, bias and peak timing). These 262 correlations were calculated for different flow regimes (all flows using the whole hydrograph, 263 rising/falling limbs using the single day differentials, and high/low flows using the top 80\% and 264 bottom 20\%). The strengths of these correlations (positive or negative) indicated which types of 265 basins (via NWM features) are benefiting most from a LSTM post-processor. Results for rising 266 limbs and falling limbs of the hydrograph were qualitatively similar to this figure, and were 267 therefore omitted.

Splitting the CAMELS catchments by calibrated / uncalibrated. Of the NWM

269 calibrated basins, 480 overlap with the 531 CAMELS catchments used in this study. In a 270 separate set of experiments, we trained the LSTM_A and the LSTM post-processors LSMT_PP 271 and LSTM_PPA) on only the 480 calibrated basins. We then used the full set of 531 catchments

272 to test the performance out-of-sample. We analyzed the 480 in-sample basins and 51 out-of-

273 sample basins separately using the NSE, bias and timing error metrics. This allowed us to 
274 determine if the LSTM is a suitable post-processing method to use in uncalibrated basins. If the

275 post-processors trained only on calibrated basins can improve streamflow predictions at

276 uncalibrated basins, then they would be considered suitable, particularly if there is no statistical

277 difference between the post-processor's performance improvement over the baseline models.

278 Sensitivity analysis and NWM process diagnostics. We trained a set of LSTM post-

279 processors using different combinations of NWM outputs as input to the LSTM, as described in

280 Table 5. To test the sensitivity to the NWM streamflow prediction itself, we trained an LSTM

281 with only streamflow (LSTM_Q_only), and excluded it from another (LSTM_PP_noQ). We

282 tested the sensitivity to the channel routing (LSTM_chrt) and land surface (LSTM_ldas)

283 components of the NWM by training LSTMs with only these dynamic inputs. These modes were

284 trained with the same specifications as the baseline LSTM_A, LSTM_PPA and LSTM_PP.

TABLE 5. Additional models for sensitivity analysis and NWM diagnostics

\begin{tabular}{lcc}
\hline & $\begin{array}{c}\text { Number of dynamic } \\
\text { LSTM parameters }\end{array}$ & Model description \\
\hline LSTM_PP_noQ & 26 & LSTM post-processor (LSTM_PP) but without streamflow or velocity. \\
LSTM_Q_only & 1 & LSTM trained with NWM streamflow only. \\
LSTM_chrt & 6 & LSTM trained with NWM channel routing outputs only. \\
LSTM_ldas & 18 & LSTM trained with NWM land surface outputs only.
\end{tabular}

Each of these models, in addition to the main post-processing models presented above,

288 have a distinct flow of information that we can use to diagnose NWM model processes. Figure 2

289 shows the information flow of each of the model subcomponents. We used the performance

290 results of the different post-processing models to assess how much information passes between

291 the model components. Nearing et al., (2015) described the method to quantify the information

292 exchange down a modeling chain (i.e., integrating over the expected effect of the conditional

293 probability), but since we used limited outputs from the NWM reanalysis, rather than the full 
294 state space, we examined the NWM only qualitatively for information loss between the major 295 NWM sub-components (land surface runoff, overland router and channel router). The LSTM 296 extracts information from its input to make predictions about its target, in our case streamflow, 297 and we assumed higher streamflow prediction accuracy indicated more information is available 298 in the NWM components used as input. If a post-processor made less accurate streamflow 299 predictions than the baseline LSTM, then this indicates that information from the atmospheric 300 forcings was lost along the NWM modeling chain.

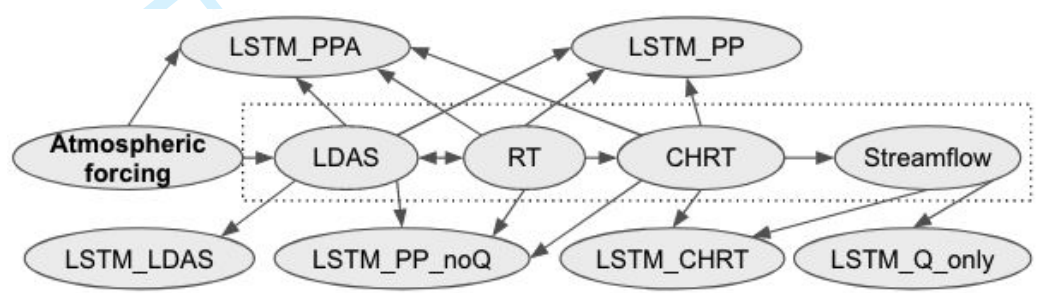

302 FIGURE 2. Process network diagram showing the information flow of each of these models. Arrows indicate the 303 information flow from one component of the model to another. The NWM components are outlined with the dashed 304 box. This is also a good guide for understanding the inputs to the different post-processing models.

\section{RESULTS}

Overall model performance

Post-processing the NWM with LSTMs significantly improved predictive performance,

308 both with or without including the atmospheric forcings as inputs into the model. Figure 3 shows

309 the cumulative distributions of three performance metrics (NSE, peak timing error, and total 310 bias). 

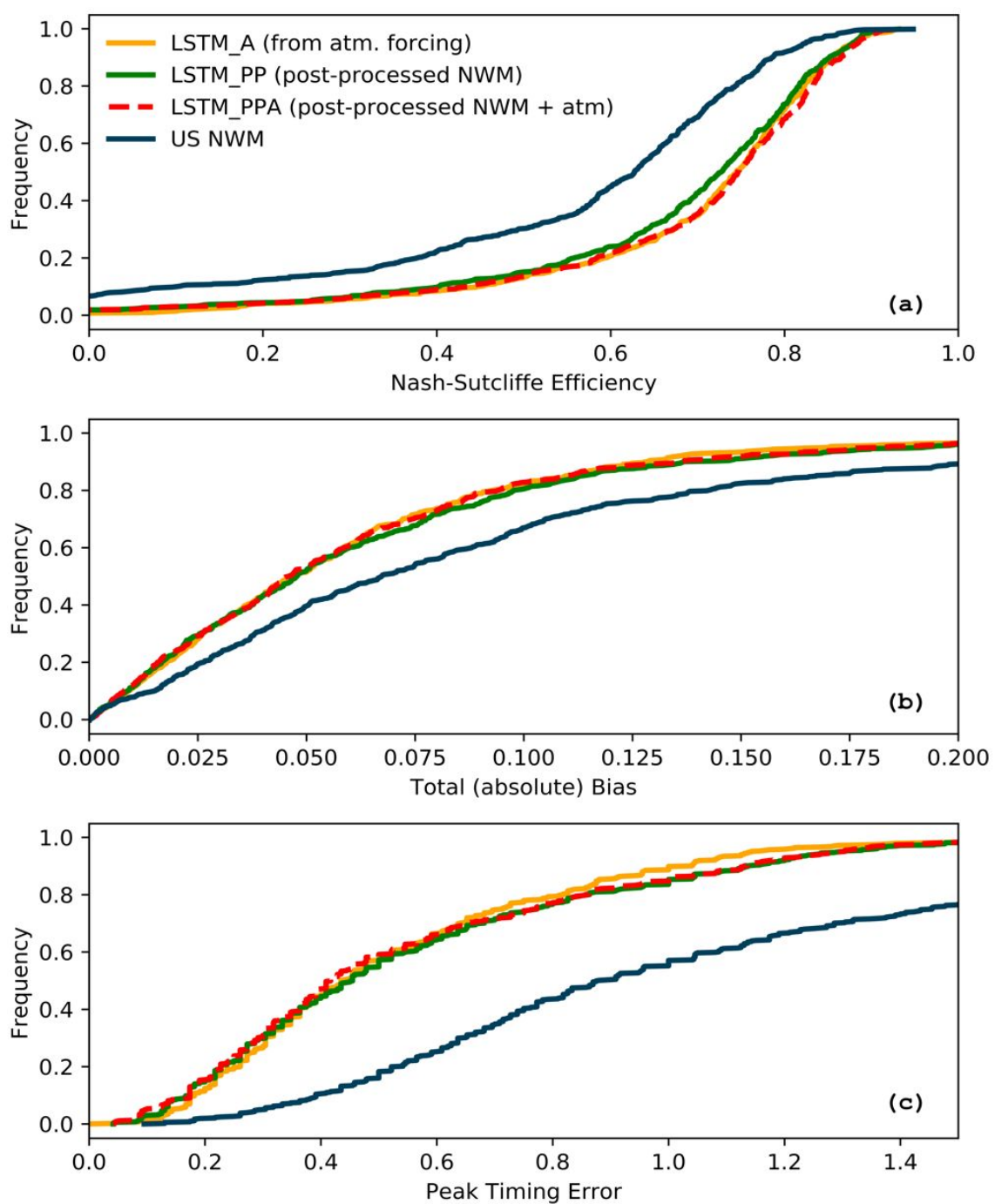

FIGURE 3. Results showing the cumulative distributions of model performance calculated as Nash-Sutcliffe

313 Efficiency (NSE), total bias, and peak timing error over a 10-year test period in 531 CAMELS catchments. The

314 National Water Model (NWM) reanalysis streamflow was averaged daily, long short-term memory (LSTM)

315 networks shown used (i) the original atmospheric inputs (LSTM_A), (ii) NWM states and fluxes only (LSTM_PP), 316 and (iii) both atmospheric forcings and NWM states and fluxes (LSTM_PPA). These figures omit the distribution tails for clarity.

The LSTM_PP improved the NSE score of the NWM mean daily streamflow at a total of $319465(88 \%)$ and reduced accuracy in 66 basins $(12 \%)$ of the total 531 CAMELS basins, improved 320 the total bias of the NWM mean daily streamflow at a total of $325(61 \%)$ of basins and improved 
321 the peak timing error at a total of 488 (92\%) of basins. The LSTM_PPA post-processor improved 322 the NSE score of the NWM mean daily streamflow at a total of $488(92 \%)$ and reduced accuracy 323 in 43 basins (8\%) of the total 531 CAMELS basins. The LSTM_PPA post-processor improved

324 the total bias of the NWM mean daily streamflow at a total of $331(62 \%)$ of basins and improved 325 the peak timing error at a total of $494(93 \%)$ of basins. The LSTM_A (the baseline LSTM 326 without NWM states and fluxes) outperformed the NWM at a total of $473(89 \%)$ and reduced 327 accuracy in 58 basins (11\%), improved the total bias of the NWM mean daily streamflow at a 328 total of 339 basins (64\%) and improved the peak timing error at a total of 484 basins $(91 \%)$. The 329 LSTM_PPA improved the greatest number of basins in terms of NSE and peak timing error and 330 the LSTM_A was the best performing model in terms of total bias. Figure 4 shows scatter plots 331 of the post-processor performance at individual basins against the performance of the baseline 332 models. 

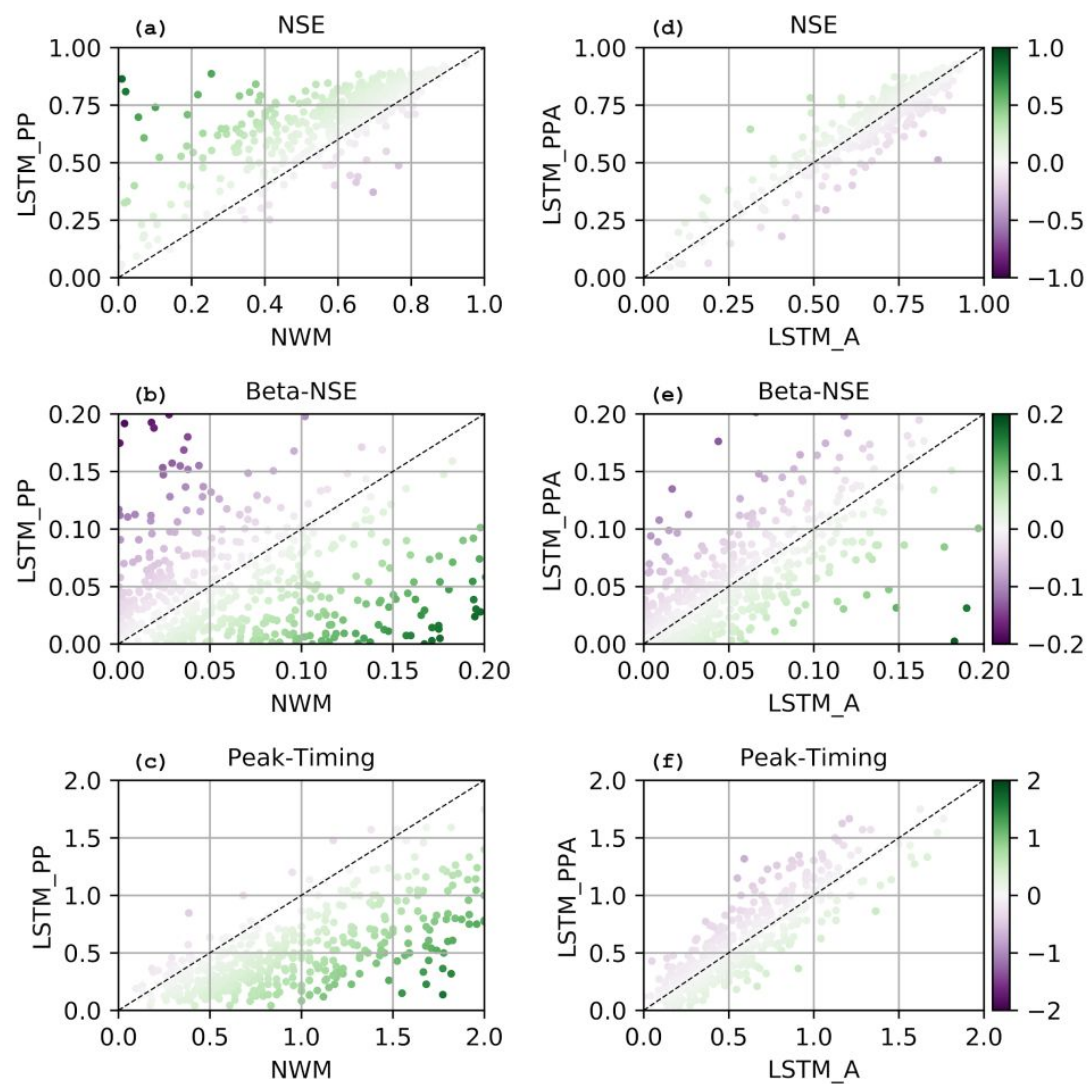

FIGURE 4. Performance differences of the post-processors against the baseline models (NWM and

335 LSTM_A) in 531 CAMELS basins across CONUS. Green indicates basins where post-processing improved 336 performance over the baseline (darker indicates larger relative improvement), and purple indicates basins where

337 there was a decrease in performance (darker indicating worse relative detriment). The first column shows the 338 performance difference between the LSTM_PP and the NWM. The second column shows the performance 339 difference between the LSTM_PPA and the LSTM_A.

341 in similar basins. The improvements of the two post-processing methods are correlated across all

342 basins $\left(r^{2}=0.995\right)$. Performance comparisons between the LSTM models and the NWM for each

343 basin are plotted spatially in Figure 5. Notice that some of the highest NSE improvements

344 between the LSTM_PP and the NWM are the worst NSE detriments between the LSTM_PPA

345 and the LSTM_A, particularly in the northern plains. This indicates that although the post- 
2

3

4

5

6

7

8

9

10

346 processor greatly improves the NWM, the information from the NWM at bad basins hinders the

347 performance of the LSTM, or in other words, the NWM passes bad information to the LSTM.

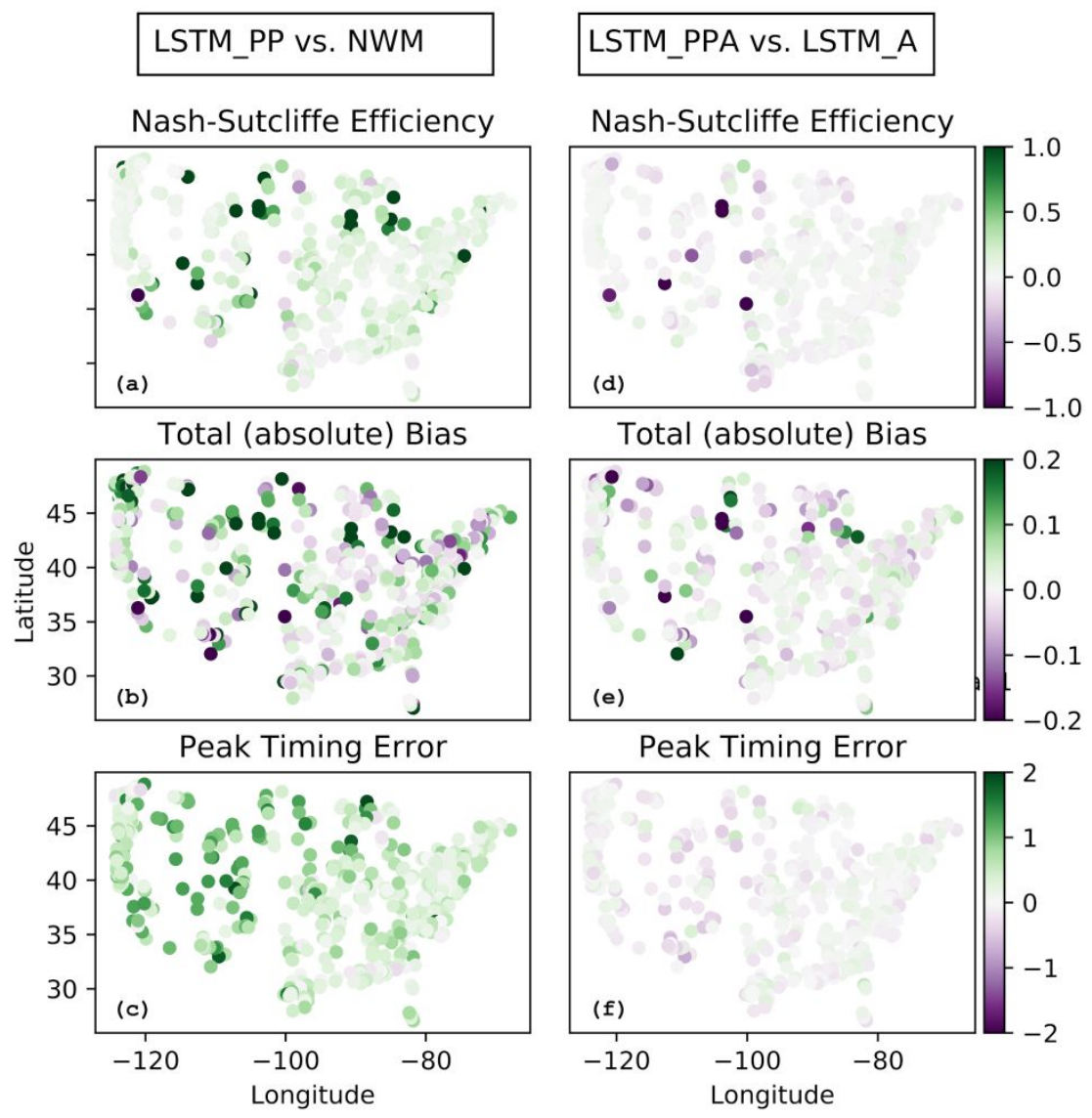

FIGURE 5. Per-basin performance change between the post-processors and baseline models (NWM and

350 LSTM_A) in 531 CAMELS basins across CONUS. Green indicates basins where post-processing improved

351 performance over the baseline (darker indicates larger relative improvement), and purple indicates basins where

352 there was a decrease in performance (darker indicating worse relative detriment). The first column (a-c) shows the

353 performance change between the LSTM_PP and the NWM. The second column (d-f) shows the performance change

354 between the LSTM_PPA and the LSTM_A. 
The LSTM post-processors improved predictive performance of the NWM according to

358 the NSE and KGE metrics, as well as their components (variance and correlation). A full set of

359 performance metrics broken down by flow regime are shown in Table 6 . The left side of the table

360 shows the average of metrics calculated individually at each basin, and the right side of the table

361 shows the metrics as calculated combining the flows from all basins. The NSE includes both

362 mean and median averages, but the rest of the metrics are only averaged by median.

TABLE 6. Predictive performance for NWM, LSTM_A and the LSTM Post-processors during various flow

regimes. The Nash-Sutcliffe Efficiency (NSE) and Kling-Gupta Efficiency (KGE) are overall performance metrics

of prediction quality. Variance, bias and correlation (R) are the components of the NSE. We calculated these in two

ways: 1) at each basin and averaged across all basins, and 2) once using the observed and predicted streamflow

values from all basins combined. Note that calculations done once across all basins do not include a test of

\begin{tabular}{|c|c|c|c|c|c|c|c|c|c|c|}
\hline Flow categories & & Calculate & d per-b & asin & & & & All basi & & \\
\hline All flows & NSE (mean) & NSE (median) & KGE & variance & bias & $\mathbf{R}$ & NSE & variance & bias & $\mathbf{R}$ \\
\hline NWM & 0.46 & 0.62 & 0.64 & 0.82 & $-0.01^{\wedge}$ & 0.82 & 0.75 & 0.85 & -0.02 & 0.87 \\
\hline LSTM_PP & $0.65^{*}$ & $0.73 *$ & $0.74 *$ & 0.86 & 0.02 & $0.87 *$ & 0.81 & 0.92 & 0.02 & 0.90 \\
\hline LSTM_A & 0.69 & 0.74 & 0.74 & 0.83 & 0.02 & 0.88 & 0.82 & 0.89 & 0.01 & 0.90 \\
\hline LSTM_PPA & 0.67 & 0.75 & 0.76 & 0.87 & 0.02 & 0.88 & 0.82 & 0.93 & 0.02 & 0.91 \\
\hline Rising limbs & NSE (mean) & NSE (median) & KGE & variance & bias & $\mathbf{R}$ & NSE & variance & bias & $\mathbf{R}$ \\
\hline NWM & 0.47 & 0.60 & 0.60 & 0.77 & -0.07 & 0.81 & 0.73 & 0.82 & -0.05 & 0.85 \\
\hline LSTM_PP & $0.64 *$ & $0.70 *$ & $0.72 *$ & $0.83 *$ & $0.00^{*}$ & $0.86^{*}$ & 0.78 & 0.88 & 0.00 & 0.88 \\
\hline LSTM_A & 0.66 & 0.71 & 0.72 & 0.80 & -0.01 & 0.86 & 0.78 & 0.85 & -0.01 & 0.88 \\
\hline LSTM_PPA & 0.65 & 0.72 & 0.74 & 0.85 & 0.00 & 0.87 & 0.79 & 0.89 & 0.00 & 0.89 \\
\hline Falling limbs & NSE (mean) & NSE (median) & KGE & variance & bias & $\mathbf{R}$ & NSE & variance & bias & $\mathbf{R}$ \\
\hline NWM & 0.29 & 0.62 & 0.64 & 0.94 & 0.03 & 0.83 & 0.78 & 0.90 & 0.00 & 0.88 \\
\hline LSTM_PP & $0.62 *$ & $0.75^{*}$ & $0.76^{*}$ & $0.95^{*}$ & 0.07 & $0.90^{*}$ & 0.87 & 0.99 & 0.04 & 0.93 \\
\hline LSTM_A & 0.69 & 0.78 & 0.77 & 0.92 & 0.05 & 0.90 & 0.87 & 0.96 & 0.03 & 0.93 \\
\hline LSTM_PPA & 0.65 & 0.77 & 0.77 & 0.94 & 0.05 & 0.90 & 0.87 & 0.98 & 0.03 & 0.93 \\
\hline
\end{tabular}

Above 80th

\begin{tabular}{ccccccccccccccc} 
percentile & NSE (mean) & NSE (median) & KGE & variance & bias & R & & NSE & variance & bias & R \\
\hline NWM & 0.17 & 0.41 & 0.54 & 0.80 & -0.13 & 0.73 & & 0.69 & 0.83 & -0.10 & 0.84
\end{tabular}

$\begin{array}{lllllllllll}\text { LSTM_PP } & 0.47^{*} & 0.57^{*} & 0.64^{*} & 0.82 & -0.08^{*} & 0.80^{*} & 0.76 & 0.89 & -0.04 & 0.90\end{array}$



nearly all flow regimes according to most metrics. The LSTM_PPA also improved upon the

Below 20th

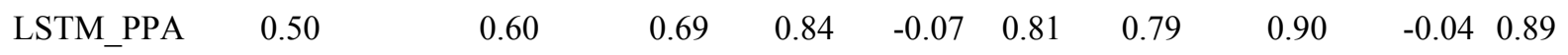

\begin{tabular}{ccccccccccccc} 
percentile & NSE (mean) & NSE (median) & KGE & variance & bias & R & & NSE & variance & bias & R \\
\hline NWM & -18384.37 & -17.47 & -1.96 & 3.79 & $1.89^{\wedge}$ & 0.36 & & 0.37 & 1.31 & 0.22 & 0.81
\end{tabular}

\begin{tabular}{rrrrrrrrrrr} 
LSTM_PP & $-6941.62^{*}$ & $-15.66^{*}$ & $-1.28^{*}$ & $2.84^{*}$ & 3.21 & $0.43^{*}$ & 0.53 & 1.30 & 0.33 & 0.90 \\
\hline LSTM_A & -4749.68 & -16.35 & -1.31 & 2.85 & 3.27 & 0.43 & 0.56 & 1.26 & 0.33 & 0.89 \\
LSTM_PPA & -5147.62 & -14.66 & -1.24 & 2.85 & 2.87 & 0.43 & 0.58 & 1.28 & 0.30 & 0.90
\end{tabular}

Note: * indicates post-processing significantly helps the NWM

Note: ${ }^{\wedge}$ indicates post-processing significantly hurts the NWM

373 limb and high flow regimes were improved by the LSTM post-processors according to every 374 metric.

375 Bias was the only metric that was reduced due to post-processing, and the difference was

376 highest in low flow regimes. Flows below the $20^{\text {th }}$ percentile were poorly predicted by all

377 models. This is likely due to the fact that all models tend to have difficulty predicting zero

378 streamflow, and the 101 basins with periods of zero streamflow affected the average

379 performance metrics. This will be discussed further in terms of hydrologic signatures.

The right side of the table has better performance values than the average of metrics

381 calculated individually at each basin. This is a result of some of the better performing basins

382 compensating for poorer performing basins, or from a different perspective, some basins have

383 relatively poor performance which weighs down the average.

\section{Performance by region}


385 Results from a regional analysis of performance are shown below in Figure 6. The 386 LSTM post-processors significantly improved the NSE over the NWM in fifteen of the eighteen 387 regions, the peak timing error in sixteen regions (all regions with enough basins for a statistical 388 evaluation) and significantly improved bias in only one region. Note that region 9 was 389 represented by only two CAMELS basins, which is not sufficient for statistical evaluation. The 390 bias was better represented by the NWM than the post-processor in five of the eighteen regions, 391 including the entire East Coast (regions 1, 2 \& 3), the Pacific Northwest (17) and the Lower392 Colorado River (15).

393 The regional performance of the LSTM post-processors and the regional performance of 394 the baseline LSTM_A were correlated with the regional performance of the NWM in terms of 395 NSE ( $r 2=0.78$ for post-processors and 0.63 for LSTM_A) and peak timing error ( $r 2=0.96$ for 396 post-processors and 0.92 for LSTM_A), but not in terms of bias $(r 2=0.24$, calculated on bias 397 although absolute bias is plotted for clarity). The post-processors and the baseline LSTM_A are 398 correlated in terms of their bias $(\mathrm{r} 2=0.91)$. A better model has a higher NSE, bias closer to zero, 399 and a lower timing error. 


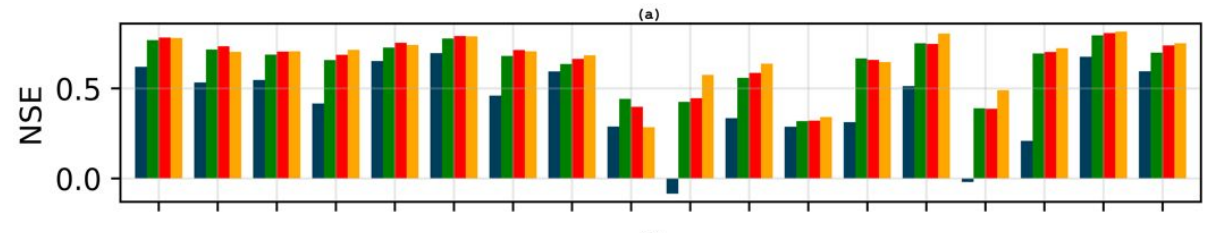

400

\section{1} 402
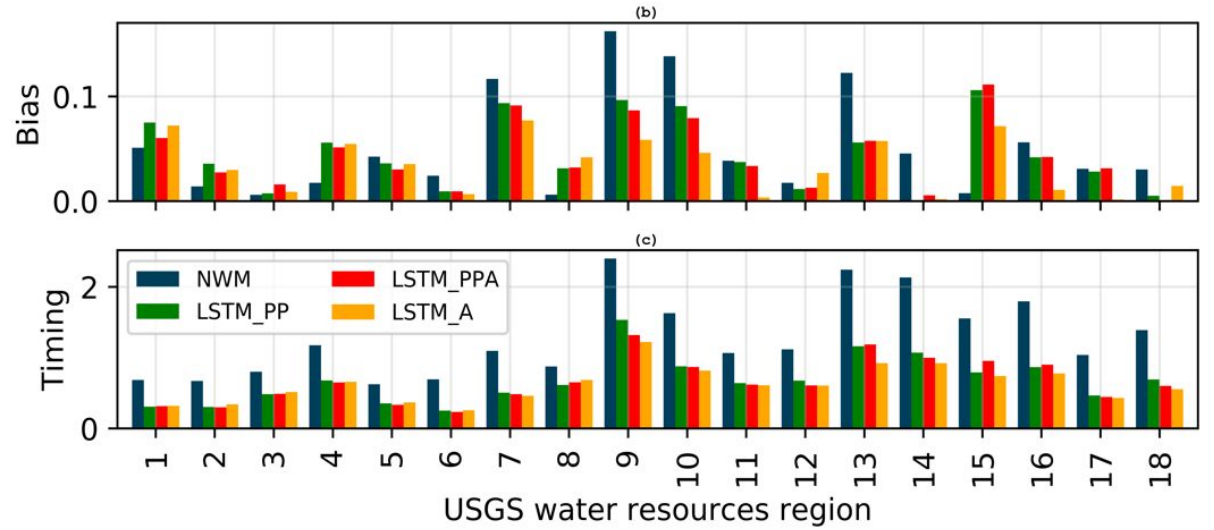

FIGURE 6. Regionally averaged performance metrics for NWM, baseline LSTM_A, and the LSTM post-processors (LSTM_PP \& LSTM_PPA) in different USGS water resources regions.

\section{Regression to predict post-processing performance improvement}

The performance of the baseline LSTM_A was more predictable than the post-

412 improvement is directly related to the performance of the NWM. 

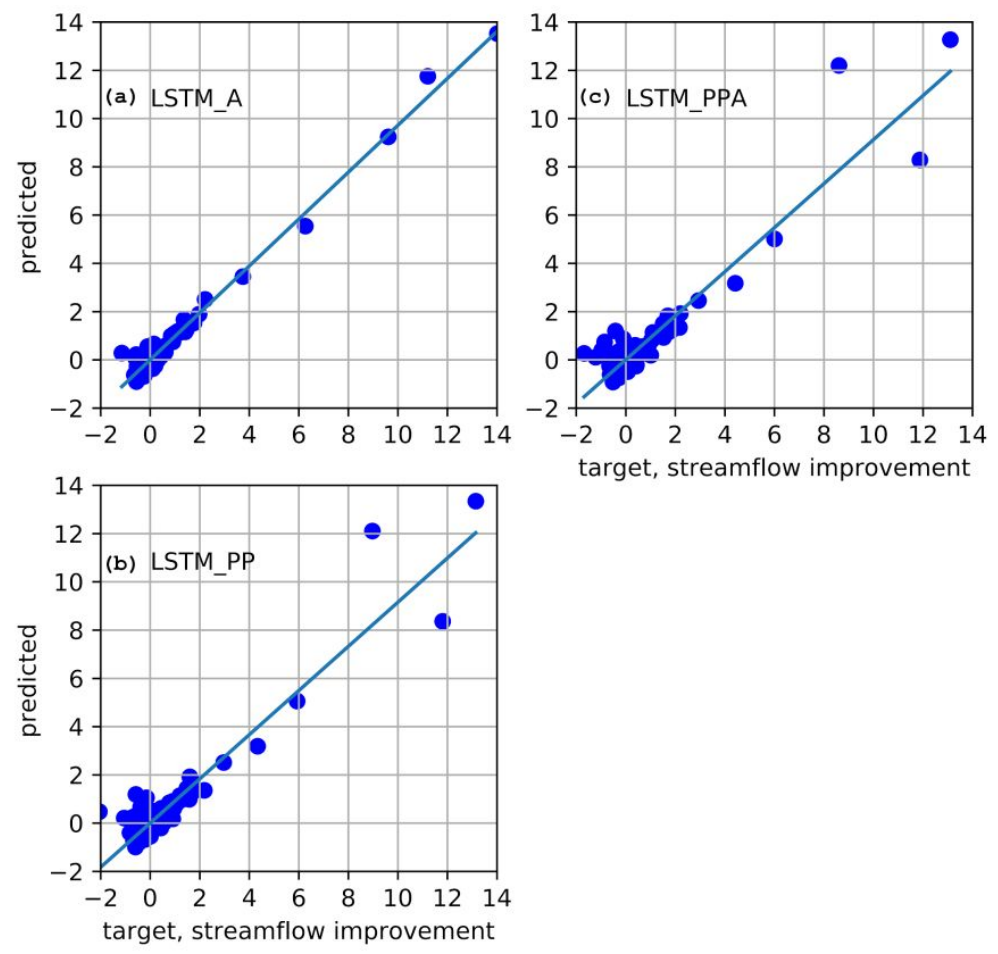

413

414 FIGURE 7. Predicting LSTM (baseline and post-processor) performance over the NWM at each basin using a linear 415 regression with NWM performance and hydrologic signatures as inputs. Scatter plots with all of the 531 basins.

416 The aim of these results is to understand whether it is possible to predict where post-

417 processing might be beneficial (remember that post-processing helped in most basins). Although

418 we found relatively high predictability in the improvement expected from post-processing, a

419 problem is that this requires knowing ahead of time the NWM performance. This prevents us

420 from predicting post-processing improvement in ungauged basins, since calculating the NWM

421 performance requires streamflow observations. The correlation analysis below may help inform

422 future efforts to learn general patters of post-processor improvement over both the NWM and the 423 baseline LSTM_A. 


\section{Correlations between NWM inputs and improvements}

Figure 8 shows correlations (over 531 basins) between the time-averaged NWM inputs

427 and changes in performance metric scores of the post-processor relative to the baseline models.

428 The LSTM_PP was compared against the NWM and the LSTM_PPA was compared against the

429 LSTM_A, although qualitatively both post-processor models were similar. The rows of this

430 figure show that correlation was weaker for differences in NSE score than total bias and peak

431 timing error. Performance differences between the NWM and the post-processor were most

432 strongly (anti)correlated with stream velocity from the channel router and accumulated

433 underground runoff from the land surface model component: basins with lower stream velocity

434 (velocity) and less underground runoff (UGDRNOFF) saw greater performance improvement

435 from (daily) post-processing. This means that in basins with high underground runoff and/or high

436 stream velocity the post-processor improvements were smaller. In contrast, basins with higher

437 total radiation (TRAD) and higher latent heat flux (LH) saw greater improvement due to post-

438 processing. This means that in basins with more radiation and heat flux the post-processor

439 improvements were larger. A direct interpretation of this could be that a flat meandering stream

440 in the Southwest will benefit from post-processing, which is consistent with the findings of Salas

441 et al. (2018) that WRF-Hydro's performance is generally poor in the Southwest. Performance

442 differences between the baseline LSTM_A and the post-processor were most strongly correlated

443 with snow water equivalent and snow depth. This is consistent with the findings of Hansen et al.

444 (2019) that the NWM represents snowpack hydrology well. 

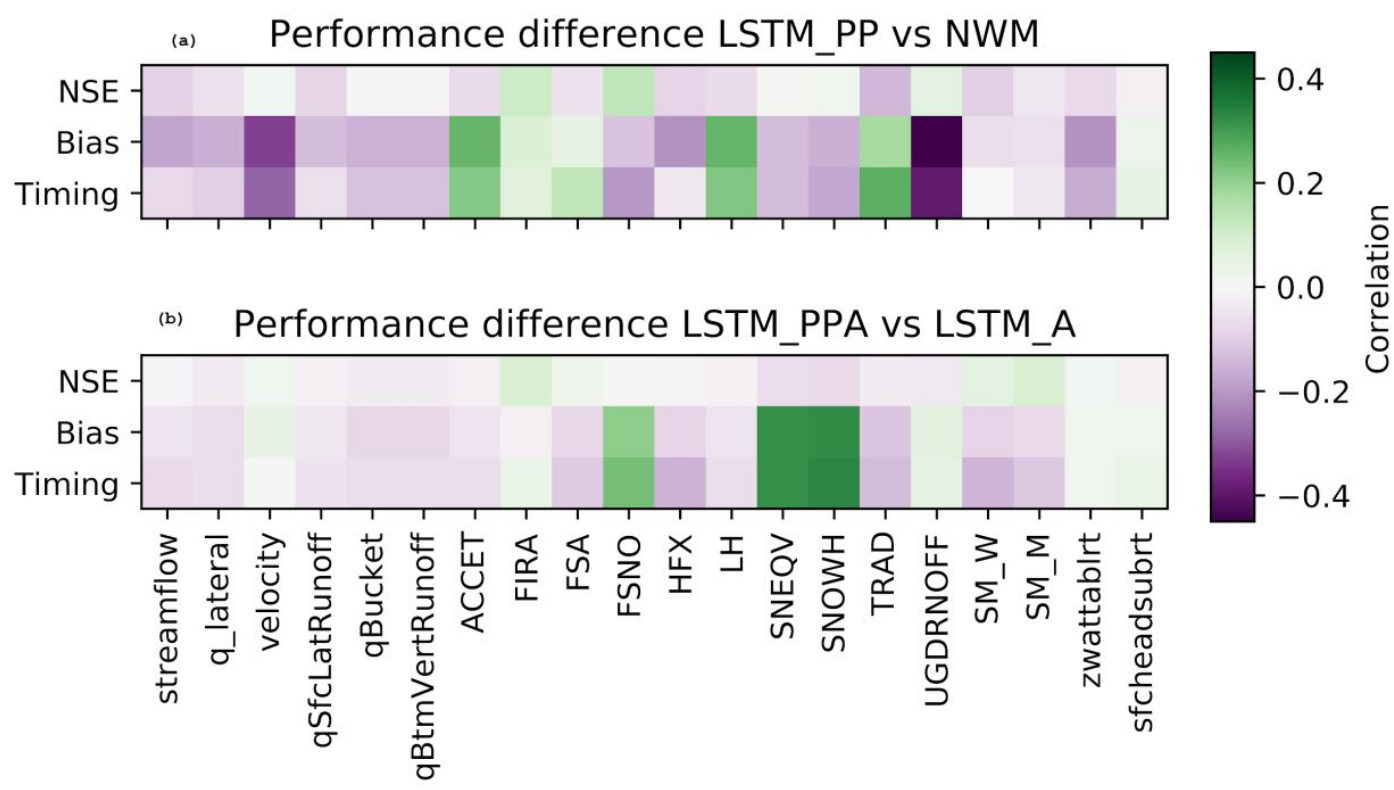

446 FIGURE 8. Correlations between the time-averaged NWM related inputs vs. performance metric differences 447 between the LSTM post-processors (LSTM_PP \& LSTM_PPA) and baseline models (NWM \& LSTM_A).

\section{$448 \quad$ Integrated gradients}

449 Figure 9 shows the relative strength of the total attribution of the dynamic inputs to the

450 LSTM_PPA averaged across the entire validation period and across basins. The ordered

451 magnitudes of the integrated gradients can be interpreted as corresponding to the order of

452 importance of inputs. The most important dynamic features for the LSTM_PPA were: (i)

453 precipitation from NLDAS, and (ii) routed streamflow from the NWM point data. Precipitation

454 inputs were weighted higher than the NWM streamflow output itself, which means that even

455 when NWM streamflow data were available, the LSTM_PPA generally learned to get

456 information directly from forcings rather than from the NWM streamflow output. This indicates

457 that the LSTM_PPA generated a new rainfall-runoff relationship rather than relying on the 
458 NWM, which is consistent with the overall results (Figure 2) that showed similar performance

459 between the LSTM_A and LSTM_PPA.

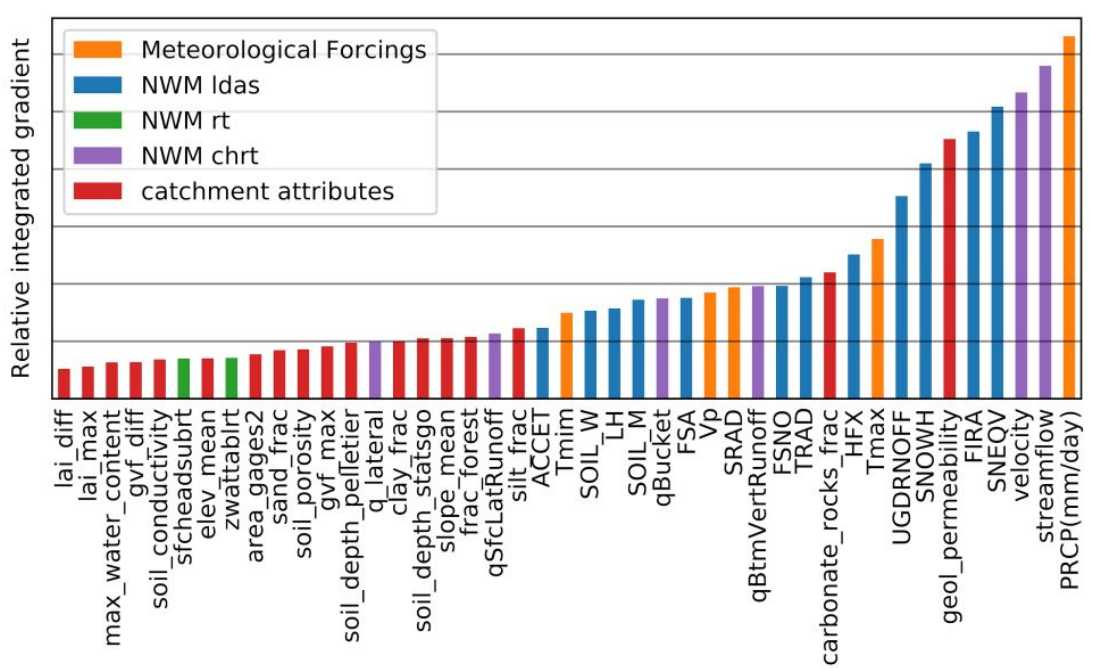

461 FIGURE 9. Attributions to the LSTM_PPA predictions. The vertical axis shows the relative magnitude of attribution 462 (importance) for each input, with precipitation (PRCP) as the top contributor and NWM-predicted runoff into 463 channel reach (q_lateral) contributing the least.

Figure 10 shows the relative strength of the total attribution of the dynamic inputs to the

465 LSTM_PP. Without the atmospheric forcings included in the post-processor inputs the NWM

466 streamflow output was by far the highest contributing dynamic input feature to the LSTM_PP.

467 The static permeability of the catchment was the next highest. 
468

469

470

\section{Representations of hydrologic signatures}

472 Results of the analysis of hydrologic signature representation are shown in Figure 11,

473 which also shows that the hydrologic signatures best represented by the NWM were similarly

474 those best represented by the LSTM_PPA. The same was true for the most poorly represented

475 hydrologic signatures in both models.

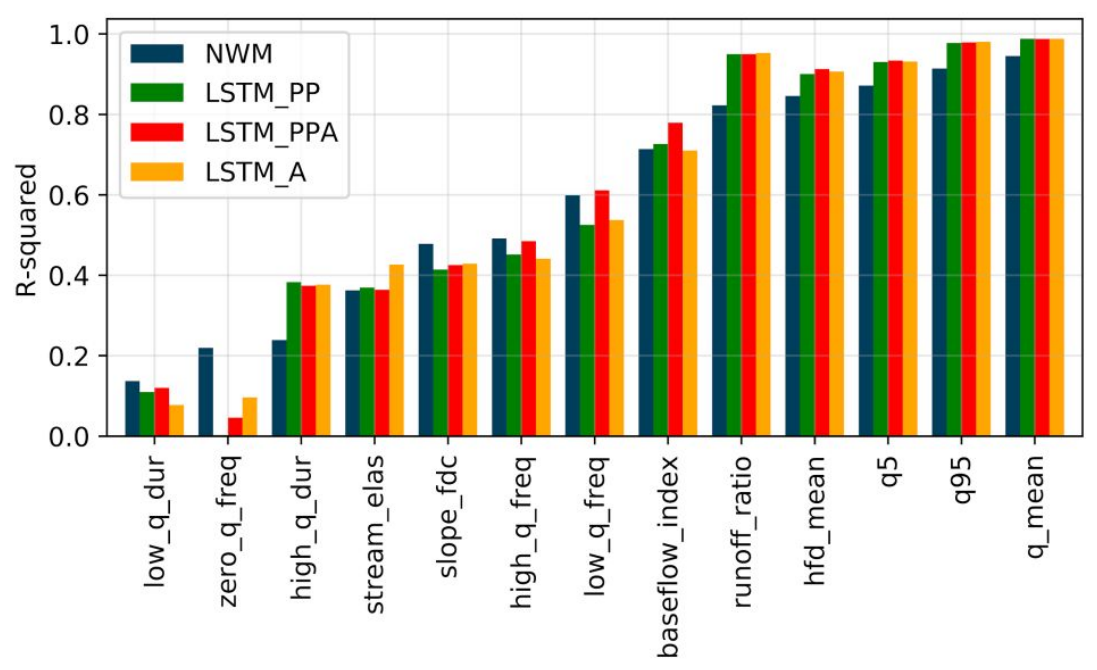


477 FIGURE 11. Correlation between simulated and observed per-basin hydrologic signatures from the NWM (blue), 478 LSTM_A (orange), LSTM_PPA (green), and LSTM_PP (red). Larger values indicates better performance.. The LSTM post-processors hurt the representation of the frequency of days with zero 480 flow. There were 101 basins with any periods of zero flow. None of these models do well 481 simulating zero flow, but the NWM is better at handling this situation, predicting zero flow 482 periods in 56 of the 101 basins. The LSTM_A, LSTM_PPA and LSTM_PP only predicted 483 periods of zero flows at 35, 29 and 25 basins, respectively. This is an important characteristic in 484 basins in the Southwest, where the NWM could use the benefit of a LSTM post-processor, so 485 this would be a good place to focus future research of theory-guided ML for hydrology.

486 The LSTM post-processor made a significant improvement over the NWM for several 487 signatures. The improvement to runoff ratio, which is the fraction of precipitation that makes it 488 through the stream gauge at the surface, could be a compensation for the uncalibrated soil 489 parameters in the NWM mentioned by Salas et al. (2018). The LSTM post-processor improved 490 both high and low flow predictions (5\% \& 95\% flow quantiles), which are important for natural 491 resources management. Mean daily discharge was the best represented hydrologic signature by 492 all models.

493 The LSTM_PPA post-processor made significant improvements over the LSTM for 494 baseflow index. This is the only signature that an LSTM post-processor improved over both the 495 NWM and the baseline LSTM_A. This signature estimates the contribution of baseflow to the 496 total discharge, which is computed by hydrograph separation. Klemeš (1986) (summarizing 497 Lindsly's Applied Hydrology) cautioned strongly against using hydrograph separation, because 498 there is no real basis for distinguishing the source of flow in a stream.

499 Results comparing gauged basins vs. ungauged basins 
Results in Table 9 summarize an analysis designed to replicate prediction in ungauged

501 basins. The table has metrics from predictions by the NWM, LSTM_A and the LSTM post-

502 processors (LSTM_PP \& LSTM_PPA) calculated only at basins that were either calibrated or

503 uncalibrated, but not both. There was no statistical difference between the calibrated and

504 uncalibrated samples. This indicates that the LSTM post-processor works in uncalibrated basins.

505 When post-processors were trained only in calibrated basins (denoted with a "C" in Table 9),

506 however, the performance in uncalibrated basins significantly deteriorated. But this is true for the

507 baseline LSTM_A as well, so it is not a result of the calibration (as calibration would not

508 influence the baseline LSTM_A), but a result of prediction at ungauged type basins. However,

509 the median performance of the post-processor predictions at ungauged type basins when trained

510 at only calibrated basins was still better significantly than the NWM in the uncalibrated basins.

511 TABLE 9. Performance of the LSTM and the LSTM post processor split between basins where the NWM was

512 calibrated vs. uncalibrated. The " $C$ " in the model name denotes that the model was trained only on calibrated basins

\begin{tabular}{|c|c|c|c|c|c|c|c|c|}
\hline \multicolumn{9}{|c|}{ Nash-Sutcliffe Efficiency } \\
\hline & \multicolumn{4}{|c|}{ Calibrated basins } & \multicolumn{4}{|c|}{ Uncalibrated basins } \\
\hline & mean & median & $\max$ & $\min$ & mean & median & $\max$ & $\min$ \\
\hline NWM & 0.49 & 0.64 & 0.95 & -10.81 & 0.18 & 0.48 & 0.79 & -7.10 \\
\hline LSTM_PP & 0.65 & 0.73 & 0.93 & -3.32 & 0.69 & 0.71 & 0.89 & 0.38 \\
\hline LSTM_A & 0.68 & 0.74 & 0.93 & -0.64 & 0.73 & 0.75 & 0.89 & 0.43 \\
\hline LSTM_PPA & 0.66 & 0.75 & 0.93 & -3.61 & 0.71 & 0.73 & 0.89 & 0.42 \\
\hline LSTM_PP(C) & 0.65 & 0.73 & 0.93 & -1.86 & 0.21 & 0.57 & 0.75 & -8.12 \\
\hline LSTM_A(C) & 0.67 & 0.74 & 0.93 & -1.13 & 0.51 & 0.67 & 0.84 & -2.54 \\
\hline LSTM_PPA(C) & 0.67 & 0.75 & 0.94 & -2.71 & 0.13 & 0.58 & 0.84 & -14.07 \\
\hline \multicolumn{9}{|c|}{ Total bias } \\
\hline & \multicolumn{4}{|c|}{ Calibrated basins } & \multicolumn{4}{|c|}{ Uncalibrated basins } \\
\hline
\end{tabular}


(

\begin{tabular}{|c|c|c|c|c|c|c|c|c|}
\hline NWM & 0.01 & -0.01 & 2.57 & -0.63 & 0.00 & -0.06 & 1.84 & -0.58 \\
\hline LSTM_PP & 0.04 & 0.02 & 1.05 & -0.24 & 0.02 & 0.01 & 0.27 & -0.12 \\
\hline LSTM_A & 0.02 & 0.02 & 0.56 & -0.22 & 0.02 & 0.01 & 0.20 & -0.11 \\
\hline LSTM_PPA & 0.03 & 0.02 & 0.98 & -0.21 & 0.01 & 0.00 & 0.22 & -0.11 \\
\hline LSTM_PP(C) & 0.01 & -0.01 & 0.92 & -0.25 & 0.06 & -0.04 & 2.15 & -0.51 \\
\hline LSTM_A(C) & 0.02 & 0.02 & 0.62 & -0.21 & 0.09 & 0.04 & 0.99 & -0.20 \\
\hline LSTM-PPA(C) & 0.01 & 0.00 & 0.95 & -0.22 & 0.06 & -0.05 & 2.89 & -0.41 \\
\hline \multicolumn{9}{|c|}{ Peak timing error } \\
\hline & \multicolumn{4}{|c|}{ Calibrated basins } & \multicolumn{4}{|c|}{ Uncalibrated basins } \\
\hline & mean & median & $\max$ & $\min$ & mean & median & $\max$ & $\min$ \\
\hline NWM & 1.06 & 0.91 & 3.00 & 0.10 & 1.04 & 0.77 & 2.70 & 0.25 \\
\hline LSTM_PP & 0.55 & 0.45 & 1.95 & 0.04 & 0.52 & 0.35 & 1.59 & 0.04 \\
\hline LSTM_A & 0.53 & 0.43 & 1.76 & 0.00 & 0.51 & 0.41 & 1.50 & 0.04 \\
\hline LSTM_PPA & 0.54 & 0.42 & 1.75 & 0.04 & 0.51 & 0.36 & 1.45 & 0.05 \\
\hline LSTM_PP(C) & 0.55 & 0.45 & 2.10 & 0.00 & 0.59 & 0.41 & 1.76 & 0.09 \\
\hline LSTM_A(C) & 0.52 & 0.43 & 1.77 & 0.00 & 0.57 & 0.50 & 1.50 & 0.08 \\
\hline LSTM_PPA(C) & 0.54 & 0.41 & 1.83 & 0.04 & 0.57 & 0.41 & 1.65 & 0.13 \\
\hline
\end{tabular}

514 The NWM, LSTM_A and the LSTM_PPA had higher NSE scores in calibrated basins

515 than the uncalibrated basins. Note that these results are from the LSTMs (baseline and post-

516 processors) trained on only basins where the NWM was calibrated. In the case of the LSTM

517 post-processors the mean NSE scores in uncalibrated basins were very low for NSE. This is a

518 result of two outlier basins (1466500, MCDONALDS BRANCH, Lat:39.9, Lon:-74.5, Area:

$5195.7 \mathrm{~km}$; and 01484100 BEAVERDAM BRANCH, Lat:38.9, Lon:-75.5, Area: 7.8km)n. Both of

520 those outlier basins are much smaller, and have lower flows, than what were included in the

521 training set. Without these basins the mean NSE scores were $0.32,0.51,0.56$ and 0.56 for the 
522 NWM, LSTM_PP, LSTM_A and LSTM_PPA, respectively. Table 9 also shows that the median

523 value of the LSTM_PPA was higher than the NWM, as was the maximum NSE value, but the

524 minimum value was exceptionally low.

$525 \quad$ The total bias in calibrated basins was generally better (lower) than the uncalibrated

526 basins. The timing error of the NWM was actually better in the uncalibrated basins, but the

527 LSTM_A and LSTM post-processors had better performance in the calibrated basins. The NSE

528 values for the NWM, LSTM_A and the LSTM post-processors (LSTM_PP and LSTM_PPA)

529 were significantly different in the calibrated basins vs. the uncalibrated basins, as were the

530 differences between the LSTM_A and LSTM post-processors (LSTM_PP and LSTM_PPA)

531 compared to the NWM. The bias values were significantly different between the two samples

532 (calibrated vs. uncalibrated), but the differences between LSTM_A and LSTM post-processors

533 vs. the NWM were not statistically different. This means that the LSTM models were successful

534 at predicting streamflow at basins outside of the calibration set.

535 LSTM post-processor sensitivity to inputs and application for process representation

536 diagnostics.

537 Figure 12 shows results from the LSTM models with inputs from different parts of the

538 NWM (land surface model only, channel router only, predicted streamflow only, and all states

539 and fluxes. The best performing LSTM models (LSTM_A \& LSTM_PPA) were the ones trained

540 with inputs that included the five atmospheric forcing variables with (LSTM_PPA) and without

541 (LSTM_A) the NWM output (these are the same models discussed in previous sections above).

542 This implies that LSTM in general was able to extract more information from the atmospheric

543 forcings than the NWM. Each of the LSTM post-processors made better average daily

544 streamflow predictions than the NWM itself, indicating that information from the atmospheric 
545 forcings is lost in the NWM model structure before the streamflow prediction is made. For

546 example, the LSTM that took as inputs only the LDAS model output from the NWM made better

547 predictions than the NWM itself, indicating that there is more information in the LDAS states

548 and fluxes than the NWM is able to translate into streamflow predictions. The same was true for

549 the states and fluxes of the CHRT component of the NWM, meaning that information is also lost

550 in the CHRT component of the NWM model structure. 

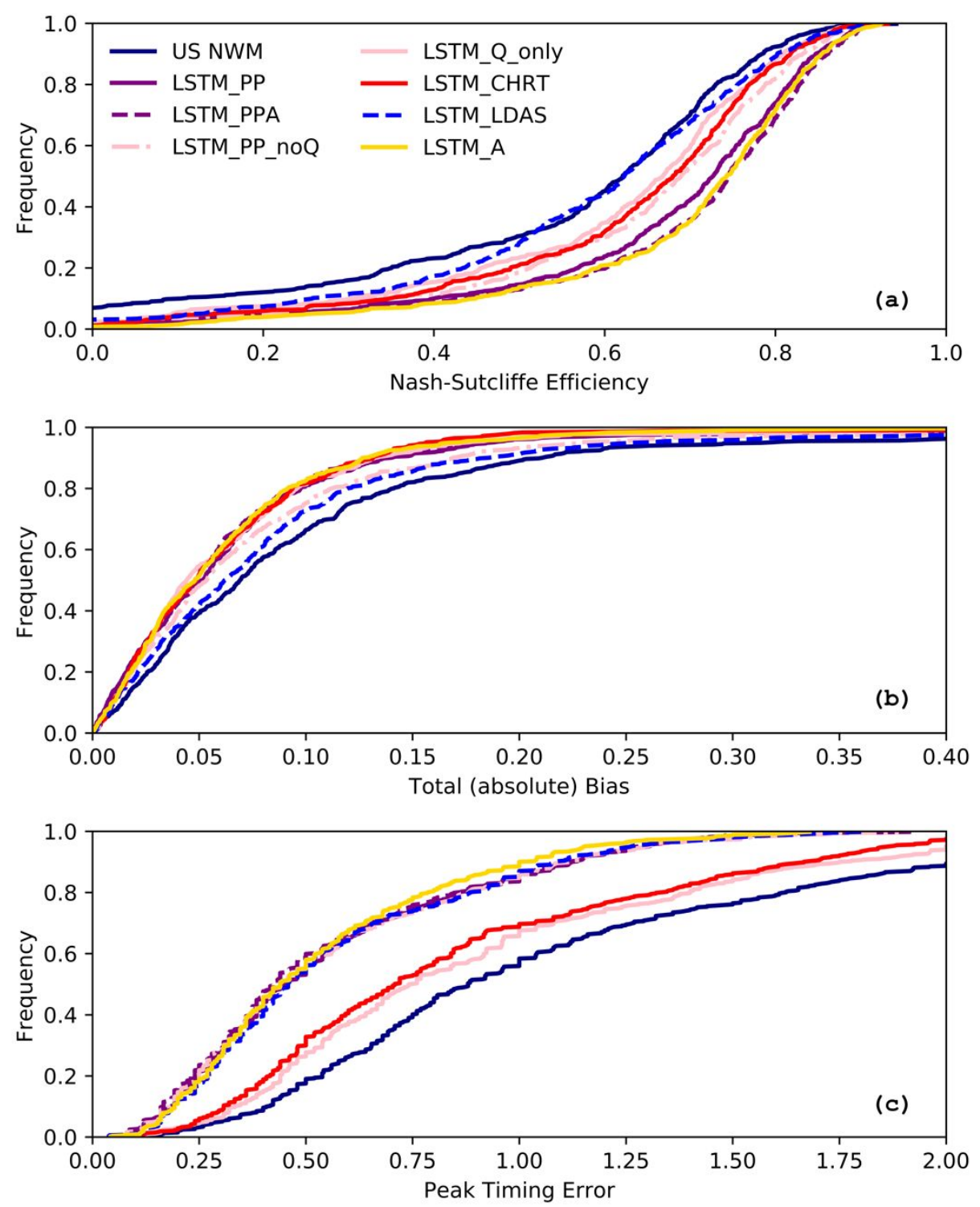

FIGURE 12. Performance of the LSTM post-processor trained with different sets of NWM output. Each of

554 these post-processors outperform the NWM. LSTM_A is the baseline LSTM trained with atmospheric forcings as

555 dynamic inputs. LSTM_PP is the NWM post-processor trained with the outputs of the NWM as dynamic inputs.

556 LSTM_PPA used both the NWM outputs and atmospheric forcings as inputs. LSTM_PP_noQ used all the NWM

557 outputs except for streamflow and velocity from the channel router. LSTM_Q_only used only streamflow from the

558 NWM output. LSTM_chrt used only the NWM channel router outputs. LSTM_ldas used only the land surface

559 fluxes as inputs. 


\section{DISCUSSION}

The baseline LSTM (LSTM_A), trained only on atmospheric forcings as dynamic inputs, 563 was better at extrapolating hydrologic conditions outside the training set than the LSTM post564 processors (LSTM_PP \& LSTM_PPA). This is shown in the analysis of prediction in ungauged 565 basins, specifically Table 9. The post-processors both failed to make reasonable predictions at 566 two basins that were much smaller than any basins included in the training set. The LSTM_A 567 was able to make good predictions in these basins. Including the NWM output as dynamic inputs 568 to the LSTM constrained the model and prevented it from learning general hydrologic 569 relationships that can be extracted to basins with characteristics that might be unrecognizable.

570 Potential for improving the performance of both the National Water Model and machine 571 learning

572 Results presented here show that the LSTM post-processors have potential to improve the 573 daily averaged flow predictions of the NWM. The LSTM post-processors provided significant 574 benefit to the NWM streamflow predictions at almost all (88\% \& 92\% for LSTM_PP \& 575 LSTM_PPA, respectively) of the 531 basins analyzed here. In the few basins where this was not 576 the case, it may be possible to use fine tuning a version of the post-processor that is specific to 577 each gauge location (as would be done in traditional model calibration), however the LSTM 578 post-processors used here can be applied to any basin, even ungauged. The post-processors were 579 trained on headwater basins, so further work would be needed to include reservoirs and other 580 management practices. It is worth noting that these LSTM models can be trained on a laptop 581 computer in a few hours, a relatively minor computational cost, and the computational cost of 
582 forward prediction is negligible. By comparison the computational cost of calibrating the NWM

583 is much higher - typically requiring HPC or cloud systems.

584 The NWM performance and the performance improvement from the LSTM post-

585 processors (LSTM_PP \& LSTM_PPA) were negatively correlated: basins with low performance 586 by the NWM have the highest performance change from the LSTM post-processors. This means 587 that post-processing can be expected to correct situations where the NWM gives bad predictions. 588 Conversely, the performance of the NWM and the LSTM_A (the baseline LSTM without NWM 589 inputs) were minimally correlated (r-squared $=0.42,0.30$ and 0.67 for NSE, bias and timing, 590 respectively). Considering also that the overall performance of the LSTM_A changed only 591 minimally from the addition of the NWM inputs (as shown in Figures 3-5 and Table 6) and that 592 the LSTM_PPA still preferred to extract more information from precipitation forcings (shown in 593 Figure 9), we might conclude that the LSTM post-processors learned new patterns of the rainfall594 runoff response, which are not fully represented by the NWM. The overall improvement in the 595 representation of hydrologic signatures indicates this new rainfall-runoff response is a better 596 representation of physical flow patterns than either the NWM or the LSTM_A. The interpretation 597 of the integrated gradient (Figures $9 \& 10$ ) and the correlations between improvement and NWM 598 features (Figure 8) indicate that this improvement of flow patterns comes from information in the 599 NWM representation of streamflow and snow states.

600 Application to real-time forecasting

601 The NWM is not simply a rainfall-runoff simulator; it simulates flow through 2.7 million 602 river reaches around CONUS, dam operations, land surface processes, hydraulics, and other 603 complications of large domain hydrology. The nature of the CAMELS catchments selected in 604 these experiments are such that they have few engineered control structures, and are under 
$60520,000 \mathrm{~km}^{2}$. The results presented in this paper show that the LSTM post-processors improved 606 streamflow predictions in the catchments studied here, which all had limited human disturbance 607 (e.g., dams, reservoirs, etc.). Kratzert et al. (2019) showed that these predictions extend into 608 ungauged basins. Our results (section "Results comparing calibrated basins vs. uncalibrated 609 basins") show that this is true for all but the poorest performing NWM basins. The immediate 610 potential for improving real-time forecasting could be deploying an LSTM for streamflow 611 prediction in undisturbed catchments, and undisturbed sub-catchments upstream of unnatural 612 hydrologic conditions such as dams, agriculture lands and urban centers. This would allow for 613 retaining conceptual representations of lakes and reservoirs that already exist in the NWM.

614 Diagnosing process-based models, physical processes and data concerns

615 The sensitivity analysis reported in Figure 12 showed that some components of the NWM 616 caused poor predictions. Specifically, information was lost in channel router (CHRT) component 617 of the model. This diagnostic method could be used to compare different schemes for future 618 versions of the NWM. For instance, changing the routing function might conserve timing 619 information from the land surface fluxes, or modifying the evapotranspiration options in Noah620 MP may conserve mass bias information from the NWM forcing engine. Such improvements 621 could be quantified with this post-processing method.

622 Each of the post-processing models tested for sensitivity (Figure 12) fall, roughly and 623 inclusively, between the NWM and the baseline LSTM_A. Based on the relative positions 624 between those bounding curves, we can identify sources of information loss through the NWM 625 modeling chain: 
626

627

628

629

630

631

632

633

634 There is potential to expand this analysis, breaking down the NWM components even further.

635 Quantification can be done with the full state space from the NWM. Retrospective runs using

636 new versions of the NWM should output the full state space for these types of analysis. This

637 diagnostics analysis using ML post-processing is possible with any physics-based, conceptual or

639 Moving forward with theory-guided machine learning

640 The post-processing procedure presented here is one of the cruder techniques currently

641 available for combining process-based and data-driven models. Several other methods of

642 combining the benefits of machine learning (predictability) with the benefits of physically

643 realistic hydrologic theory (robustness) are in development. For example, Pelissier et al. (2019)

644 integrated a trained Gaussian Processes into the state-space dynamics of a process-based land

645 surface model for predicting soil moisture time series. Another example is using physical

646 principles to constrain the loss function of an ML model during training - for example Hoedt et

647 al. (2020) integrated mass balance constraints into an LSTM and applied this model to the same

648531 basins used in this study. Implementing post-processing is relatively straightforward 
649 compared to other techniques such as adding physics into ML code or using ML to dynamically 650 update the state variables.

651 Using ML for post-processing has potential for advancing the explainability of data652 driven models. We showed that the LSTM model representation of hydrologic signatures (post653 processed and baseline) is highly correlated with the NWM. This indicates that the "learned" 654 functions mapping inputs to streamflow are actually quite similar. We might have trouble 655 expressing the "learned" LSTM with compact formulas (e.g., PDEs), given the high number of 656 trained model weights, but we can use them with confidence knowing their structural similarities 657 with process-based models like the NWM.

\section{CONCLUSION}

The LSTM post-processors (LSTM_PPA \& LSTM_PP) significantly outperformed the

660 NWM, but only slightly outperformed the LSTM_A (the baseline LSTM without the NWM 661 states and fluxes as inputs). LSTMs, in general, are capable of learning the dynamics of rainfall662 runoff processes, gaining little additional information from the conceptualizations coded within 663 the NWM. The "pure" post-processing model (LSTM_PP) outperformed the NWM in terms of 664 bias, and significantly outperformed the NWM in terms of NSE and timing. A decision to use the 665 LSTM as a post-processor for the NWM should be made with professional judgement, 666 considering the comparison of the NWM, LSTM and LSTM post-processor's performance. In 667 locations where the NWM is not calibrated, or the hydrologic conditions are not well understood, 668 it would be best to use the LSTM without the influence from the NWM.

669 The results indicate that there is more information in the atmospheric forcings about 670 streamflow observations than in the NWM outputs, including the NWM streamflow prediction. 
671 The NWM loses information between the atmospheric forcing inputs and the outputs. The NWM

672 land surface component (LDAS) loses information about mass conservation (shown from the

673 bias error), and the channel router (CHRT) loses information about streamflow timing. The

674 NWM routing scheme should be considered as a priority for improving the NWM.

675

DATA AVAILABILITY

676 All data and code used in this paper are publicly available in the following locations:

677 U.S. National Water Model: https://docs.opendata.aws/nwm-archive/readme.html

678 CAMELS data: https://ral.ucar.edu/solutions/products/camels

679 Data processing code: https://github.com/jmframe/nwm-reanalysis-model-data-processing, 680 DOI: $10.5281 /$ zenodo.4642605

681 LSTM code: https://github.com/kratzert/ealstm_regional_modeling

682 Post-processing and analysis code: https://github.com/jmframe/nwm-post-processing-with683 1stm, DOI: 10.5281/zenodo.4642603

\section{ACKNOWLEDGEMENTS}

686 Authors from Johannes Kepler University were partially supported by a Google faculty research 687 award. Jonathan Frame from the University of Alabama supported by the NASA Terrestrial 688 Hydrology Program. Grey Nearing (then at the University of Alabama) was partially supported 689 by the NCAR COMET program on a cooperative award with the National Water Center. The 690 LSTM models presented here were trained using computational resources from the NASA

691 Center for Climate Simulation. We thank the reviewers for their valuable feedback and 692 constructive criticism throughout the review of this paper.

\section{LITERATURE CITED}


694 Addor, N., A.J. Newman, N. Mizukami, and M. P. Clark, 2017. The CAMELS Data Set:

695 Catchment Attributes and Meteorology for Large-Sample Studies. Earth Syst. Sci 21:

696 5293-5313. https://doi.org/10.5194/hess-21-5293-2017.

697 Addor, N., G. Nearing, C. Prieto, A. J. Newman, N. Le Vine, and M. P. Clark, 2018. A

698 Ranking of Hydrological Signatures Based on Their Predictability in Space. Water

699 Resources Research 54, no. 11: 8792-8812. https://doi.org/10.1029/2018WR022606.

700 Chadalawada, J., H. M.V.V. Herath, and V. Babovic, 2020. Hydrologically Informed

701

Machine Learning for Rainfall-Runoff Modeling: A Genetic Programming-Based

702

Toolkit for Automatic Model Induction. Water Resources Research 56, no. 4: 1-23.

703 https://doi.org/10.1029/2019WR026933.

704

Cosgrove, B, D. Gochis, E. P. Clark, Z. Cui, A. L. Dugger, G. M. Fall, X. Feng, M. A.

705

Fresch, J. J. Gourley, S. Khan, D. Kitzmiller, H. S. Lee, Y. Liu, J. L. McCreight, A. J.

706

Newman, A. Oubeidillah, L. Pan, C. Pham, F. Salas, K. M. Sampson, M. Smith, G.

707

Sood, A. Wood, D. N. Yates, W. Yu and Y. Zhang, 2015. Hydrologic Modeling at the

708

National Water Center: Operational Implementation of the WRF-Hydro Model to

709

Support National Weather Service Hydrology. In AGU Fall Meeting Abstracts.

710 Daw, A., R. Q. Thomas, C. C. Carey, J. S. Read, A. P. Appling, and Anuj Karpatne, 2020.

Physics-Guided Architecture (PGA) of Neural Networks for Quantifying Uncertainty

712 in Lake Temperature Modeling. Proceedings of the 2020 SIAM International

713 Conference on Data Mining, 532-40. https://doi.org/10.1137/1.9781611976236.60. 
714 Elmer, N. J, 2019. Using Satellite Observations of River Height and Vegetation To Improve 715 National Water Model Initialization and Streamflow Prediction. PhD diss., The $716 \quad$ University of Alabama in Huntsville.

717 Gauch, M., J. Mai, and J. Lin, 2019. The Proper Care and Feeding of CAMELS: How 718 Limited Training Data Affects Streamflow Prediction 2342: 0-2. $719 \quad$ http://arxiv.org/abs/1911.07249.

720 Gauch, Martin, Frederik Kratzert, Daniel Klotz, Grey Nearing, Jimmy Lin, and Sepp $721 \quad$ Hochreiter. 2020. Rainfall-Runoff Prediction at Multiple Timescales with a Single 722 Long Short-Term Memory Network, 2020, 1-24. http://arxiv.org/abs/2010.07921.

723 Gupta, H. V., T. Wagener, and Y. Liu, 2008. Reconciling Theory with Observations: 724 Elements of a Diagnostic Approach to Model Evaluation. Hydrological Processes 7252274 , no. November 2008: 2267-74. https://doi.org/10.1002/hyp.6989.

726 Gupta, H. V., H. Kling, K. K. Yilmaz, and G. F. Martinez, 2009. Decomposition of the $727 \quad$ Mean Squared Error and NSE Performance Criteria: Implications for Improving $728 \quad$ Hydrological Modelling. Journal of Hydrology 377, no. 1-2: 80-91. $729 \quad$ https://doi.org/10.1016/j.jhydrol.2009.08.003.

730 Hansen, C., J. S. Shiva, S. McDonald, and A. Nabors, 2019. Assessing Retrospective 731 National Water Model Streamflow with Respect to Droughts and Low Flows in the 732 Colorado River Basin. Journal of the American Water Resources Association 55, no. 4: 733 964-75. https://doi.org/10.1111/1752-1688.12784. 
734 Hochreiter, S, 1991. "Untersuchungen Zu Dynamischen Neuronalen Netzen.” Doctoral 735 diss., Institut Für Informatik, Technische Universität, Munchen 736 http://people.idsia.ch/ juergen/SeppHochreiter1991ThesisAdvisorSchmidhuber.pdf.

737 Hochreiter, S., and J. Schmidhuber, 1997. Long Short-Term Memory. Neural Computation 7389 , no. 8: 1735-80. https://doi.org/10.1162/neco.1997.9.8.1735.

739 Hoedt, P. J., F. Kratzert, D. Klotz, C. Halmich, M. Holzleitner, G. Nearing, S. Hochreiter, 740 and G. Klambauer. 2021. MC-LSTM: Mass-Conserving LSTM, 2021, 1-32.

$741 \quad$ http://arxiv.org/abs/2101.05186.

742 Karpatne, A., W. Watkins, J. Read, and V. Kumar, 2017a. Physics-Guided Neural Networks 743 (PGNN): An Application in Lake Temperature Modeling. $744 \quad$ http://arxiv.org/abs/1710.11431.

745 Karpatne, A., G. Atluri, J. H. Faghmous, M. Steinbach, A. Banerjee, A. Ganguly, S.

746 Shekhar, N. Samatova, and V. Kumar, 2017b. Theory-Guided Data Science: A New

747 Paradigm for Scientific Discovery from Data. IEEE Transactions on Knowledge and 748 Data Engineering 29, no. 10: 2318-31. https://doi.org/10.1109/TKDE.2017.2720168.

749 Kim, J., L. Read, L. E. Johnson, D. Gochis, R. Cifelli, and H. Han, 2020. An Experiment on 750 Reservoir Representation Schemes to Improve Hydrologic Prediction: Coupling the 751 National Water Model with the HEC-ResSim. Hydrological Sciences Journal 0, no. 0: 752 1. https://doi.org/10.1080/02626667.2020.1757677.

753 Klemeš, V, 1986. Dilettantism in Hydrology: Transition or Destiny? Water Resources 754 Research 22, no. 9 S: 177S-188S. https://doi.org/10.1029/WR022i09Sp0177S. 
755 Kratzert, F., D. Klotz, C. Brenner, K. Schulz, and M. Herrnegger, 2018. Rainfall-Runoff

756 Modelling Using Long Short-Term Memory (LSTM) Networks. Hydrology and Earth

757 System Sciences 22, no. 11: 6005-22. https://doi.org/10.5194/hess-22-6005-2018.

758 Kratzert, F., D. Klotz, M. Herrnegger, A. K. Sampson, S. Hochreiter, and G. S. Nearing, 759 2019a. Towards Improved Predictions in Ungauged Basins: Exploiting the Power of

760 Machine Learning. Water Resources Research, 2019WR026065.

$761 \quad$ https://doi.org/10.1029/2019WR026065.

762 Kratzert, F., D. Klotz, G. Shalev, G. Klambauer, S. Hochreiter, and G. S. Nearing, 2019b.

763 Towards Learning Universal, Regional, and Local Hydrological Behaviors via

764 Machine Learning Applied to Large-Sample Datasets. Hydrology and Earth System

765 Sciences 23, no. 12: 5089-5110. https://doi.org/10.5194/hess-23-5089-2019.

766 Nearing, G. S., Benjamin L. Ruddell, Martyn P. Clark, Bart Nijssen, and Christa Peters-

767 Lidard. "Benchmarking and Process Diagnostics of Land Models.” Journal of

768 Hydrometeorology, 2018, JHM-D-17-0209.1. https://doi.org/10.1175/JHM-D-17-

$769 \quad 0209.1$.

770 Nearing, G. S., F. Kratzert, D. Klotz, P.J. Hoedt, G. Klambauer, S. Hochreiter, H. Gupta, S.

771 Nevo, and Y. Matias. 2020. A Deep Learning Architecture for Conservative

772 Dynamical Systems: Application to Rainfall-Runoff Modeling. AI for Earth Sciences

$773 \quad$ Workshop at NEURIPS 2020, 2020.

774 Newman, A. J., M. P. Clark, K. Sampson, A. Wood, L. E. Hay, A. Bock, R. J. Viger, D.

775 Blodgett, L. Brekke, J. R. Arnold, T. Hopson, and Q. Duan, 2015. Development of a

776 Large-Sample Watershed-Scale Hydrometeorological Data Set for the Contiguous 
777 USA: Data Set Characteristics and Assessment of Regional Variability in Hydrologic 778 Model Performance. Hydrology and Earth System Sciences 19, no. 1: 209-23.

779 https://doi.org/10.5194/hess-19-209-2015.

780 National Oceanic and Atmospheric Administration (NOAA). 2019. Stakeholder

781 Engagement to Inform National Weather Service Hydrologic Products and Services to 782 Meet User Needs NOAA National Weather Service Water Resources Services Branch and Office of Water Prediction. https://www.weather.gov/media/water/StakeholderEngagementtoInformNWMProducts Services2019.pdf

Pelissier, C., J. Frame, and G. Nearing, 2019. Combining Parametric Land Surface Models with Machine Learning. arXiv preprint arXiv:2002.06141.

Read, J. S., Jia, X., Willard, J., Appling, A. P., Zwart, J. A., Oliver, S. K., et al. 2019. Process-guided deep learning predictions of lake water temperature. Water Resources Research, 55, 9173- 9190. https://doi.org/10.1029/2019WR024922 Deep Learning and Process Understanding for Data-Driven Earth System Science. Nature 566: 195-204. https://doi.org/10.1038/s41586-019-0912-1.

794 Salas, F. R., M. A. Somos-Valenzuela, A. Dugger, D. R. Maidment, D. J. Gochis, C. H. David, W. Yu, D. Ding, E. P. Clark, and N. Noman, 2018. Towards Real-Time 797 Continental Scale Streamflow Simulation in Continuous and Discrete Space. Journal of the American Water Resources Association 54, no. 1: 7-27.

798 https://doi.org/10.1111/1752-1688.12586. 
799 Steiger, J., and M. Browne, 1984. The Comparison of Interdependent Correlations between 800 Optimal Linear Composites. Psychometrika 49, no. 1: 11-24.

$801 \quad$ https://doi.org/10.1017/CBO9781107415324.004.

802 Sundararajan, M., A. Taly, and Q. Yan, 2017. Axiomatic Attribution for Deep Networks. 803 34th International Conference on Machine Learning, ICML 2017 7: 5109-18.

804 Tartakovsky, A. M., C. O. Marrero, P. Perdikaris, G. D. Tartakovsky, and D. Barajas805 Solano, 2020. Physics-Informed Deep Neural Networks for Learning Parameters and 806 Constitutive Relationships in Subsurface Flow Problems. Water Resources Research $807 \quad 56$, no. 5: 1-16. https://doi.org/10.1029/2019WR026731.

808 Xia, Y., K. Mitchell, M. Ek, J. Sheffield, B. Cosgrove, E. Wood, L. Luo, C. Alonge, H. 809 Wei, J. Meng, B. Livneh, D. Lettenmaier, V. Koren, Q. Duan, K. Mo, Y. Fan, and D. 810 Mocko, 2012. Continental-Scale Water and Energy Flux Analysis and Validation for 811 the North American Land Data Assimilation System Project Phase 2 (NLDAS-2): 1. 812 Intercomparison and Application of Model Products. Journal of Geophysical Research $813 \quad$ Atmospheres 117, no. 3. https://doi.org/10.1029/2011JD016048.

814 Ye, A., Q. Duan, X. Yuan, E. F Wood, and J. Schaake, 2014. Hydrologic Post-Processing 815 of MOPEX Streamflow Simulations. Journal of Hydrology 508: 147-56. $816 \quad$ https://doi.org/10.1016/j.jhydrol.2013.10.055. 